\title{
Boundary estimates for the elastic wave equation in almost incompressible materials*
}

\author{
Heinz-Otto Kreiss ${ }^{\dagger}$ and N. Anders Petersson ${ }^{\ddagger}$
}

May 30, 2022

\begin{abstract}
We study the half-plane problem for the elastic wave equation subject to a free surface boundary condition, with particular emphasis on almost incompressible materials. A normal mode analysis is developed to estimate the solution in terms of the boundary data, showing that the problem is boundary stable. The dependence on the material properties, which is difficult to analyze by the energy method, is made transparent by our estimates. The normal mode technique is used to analyze the influence of truncation errors in a finite difference approximation. Our analysis explains why the number of grid points per wave length must be increased when the shear modulus $(\mu)$ becomes small, that is, for almost incompressible materials. To obtain a fixed error in the phase velocity of Rayleigh surface waves as $\mu \rightarrow 0$, our analysis predicts that the grid size must be proportional to $\mu^{1 / 2}$ for a second order method. For a fourth order method, the grid size can be proportional to $\mu^{1 / 4}$. Numerical experiments confirm these scalings and illustrate the superior efficiency of the fourth order method.
\end{abstract}

\section{Introduction}

Consider the half-plane problem for the two-dimensional elastic wave equation in a homogeneous isotropic material. By scaling time to give unit density, the displacement with

${ }^{*}$ This work was performed under the auspices of the U.S. Department of Energy by Lawrence Livermore National Laboratory under Contract DE-AC52-07NA27344.

${ }^{\dagger}$ Träskö-Storö Institute of Mathematics, Stockholm, Sweden.

${ }^{\ddagger}$ Corresponding author. Center for Applied Scientific Computing, Lawrence Livermore National Laboratory, P.O. Box 808, Livermore, CA 94551, E-mail: andersp@llnl.gov. 
Cartesian components $(u, v)^{T}$ is governed by

$$
\left\{\begin{array}{l}
u_{t t}=\mu \Delta u+(\lambda+\mu)\left(u_{x}+v_{y}\right)_{x}+F_{1}(x, y, t), \\
v_{t t}=\mu \Delta v+(\lambda+\mu)\left(u_{x}+v_{y}\right)_{y}+F_{2}(x, y, t)
\end{array} \quad x \geq 0,-\infty<y<\infty, t \geq 0,\right.
$$

where $\left(F_{1}, F_{2}\right)^{T}$ is the internal forcing. Here, $\lambda$ and $\mu>0$ are the first and second Lamé parameters of the material. We assume that both parameters are constant and $\lambda>0$. The displacement is subject to initial conditions

$$
\left\{\begin{array} { l } 
{ u ( x , y , 0 ) = f _ { 1 0 } ( x , y ) , } \\
{ u _ { t } ( x , y , 0 ) = f _ { 2 0 } ( x , y ) , }
\end{array} \quad \left\{\begin{array}{l}
v(x, y, 0)=f_{11}(x, y), \\
v_{t}(x, y, 0)=f_{21}(x, y),
\end{array} \quad x \geq 0,-\infty<y<\infty .\right.\right.
$$

In this paper we consider normal stress boundary conditions along the $x=0$ boundary,

$$
\left\{\begin{array}{l}
u_{x}+\gamma^{2} v_{y}=g_{1}(y, t), \\
u_{y}+v_{x}=g_{2}(y, t),
\end{array} \quad x=0,-\infty<y<\infty, t \geq 0,\right.
$$

where $g_{1}$ and $g_{2}$ are boundary forcing functions, and

$$
\gamma^{2}=\frac{\lambda}{2 \mu+\lambda}
$$

When $g_{1}=0$ and $g_{2}=0$, (3) is called a free surface boundary condition.

Since time was scaled to give unit density, the elastic energy is given by

$$
E(t)=\frac{1}{2} \int_{-\infty}^{\infty} \int_{0}^{\infty}\left(u_{t}^{2}+v_{t}^{2}\right)+\lambda\left(u_{x}+v_{y}\right)^{2}+\mu\left(2 u_{x}^{2}+2 v_{y}^{2}+\left(u_{y}+v_{x}\right)^{2}\right) d x d y
$$

It is well known (see e.g. Achenbach [1], pp. 59-61) that the elastic energy satisfies

$$
\begin{aligned}
\frac{d}{d t} E(t)=\int_{-\infty}^{\infty} \int_{0}^{\infty}\left(u_{t} F_{1}+v_{t} F_{2}\right) & d x d y \\
& -\left.\int_{-\infty}^{\infty}\left(u_{t}\left((2 \mu+\lambda) u_{x}+\lambda v_{y}\right)+v_{t} \mu\left(u_{y}+v_{x}\right)\right)\right|_{x=0} d y
\end{aligned}
$$

In particular, without boundary and interior forcing, the elastic energy is conserved,

$$
E(t)=E(0), \quad t>0, \quad g_{1}=g_{2}=0, F_{1}=F_{2}=0
$$

Note that the elastic energy is a semi-norm of the solution. The energy estimate bounds this semi-norm in terms of the initial data and the internal forcing $\left(F_{1}, F_{2}\right)^{T}$. For this reason, the elastic wave equation is a well-posed problem. However, the energy estimate does 

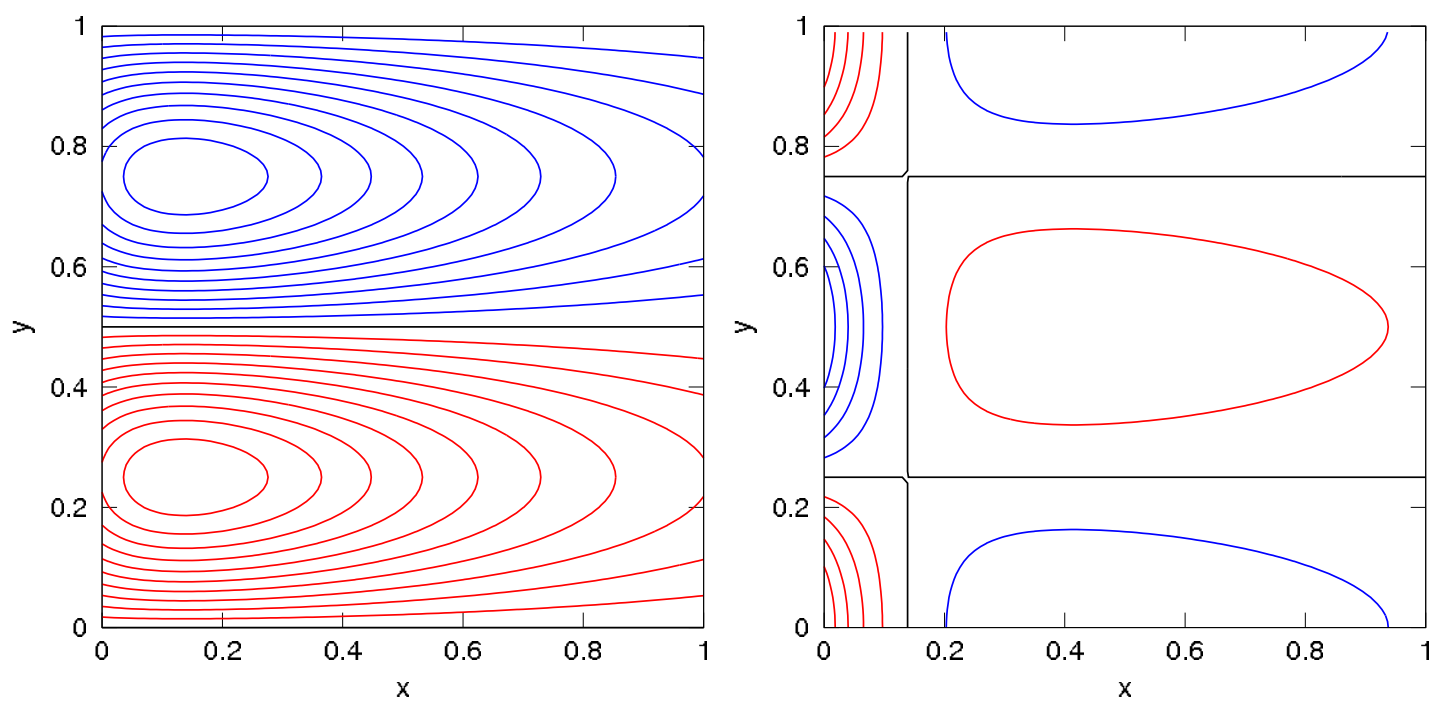

Figure 1: A contour plot of a Rayleigh surface wave as function of $(x, y)$ at $t=0$ for a material with $\lambda=1$ and $\mu=0.01$. The $u$-component is shown to the left and the $v$ component to the right. The contour levels are given between -0.5 and 0.5 , with spacing 0.05. Red and blue lines correspond to negative and positive values, respectively. The zero level is plotted in black.

not provide detailed insight into how the solution depends on the material parameters, or the boundary data.

The material parameters, in particular the ratio $\mu / \lambda$, strongly influences the accuracy of numerical solutions of the elastic wave equation. As a motivating example, we propagate a Rayleigh surface wave using a second order accurate finite difference method. In the numerical experiment, we make the $y$-direction 1-periodic and take the wave length to be one. A free surface boundary condition is imposed at $x=0$. The Rayleigh surface wave propagates harmonically in the $y$-direction and decays exponentially in $x$, see Figure1. We take $\lambda=1$ and vary $\mu$, which gives the surface wave a phase velocity that is proportional to $\sqrt{\mu}$. We discretize the elastic wave equation on a grid with grid size $h$, corresponding to $P=1 / h$ grid points per wave length; further details of this numerical experiment are presented in $\S 5$. In Figure 2, we report the error in the numerical solution at time $t=20$. For the smaller values of $\mu$, a large number of grid points per wave length are needed to obtain an acceptable error level and a second order convergence rate. For the finest mesh with 200 grid points per wave length, the error increases by more than an order of magnitude (from $3.76 \cdot 10^{-3}$ to $5.09 \cdot 10^{-2}$ ), when $\mu$ decreases by two orders of magnitude (from $10^{-1}$ to $10^{-3}$ ). Note that the gradient of the exact solution only depends weakly on $\mu$ and is of the order $\mathcal{O}(1 / 2 \pi)$ for all values of $\mu>0$. Hence, the loss of accuracy is not due to poor resolution in space. Furthermore, the phase velocity of the surface wave becomes slower and slower as $\mu \rightarrow 0$, while the time step is governed by $\sqrt{\lambda+3 \mu}$, which 


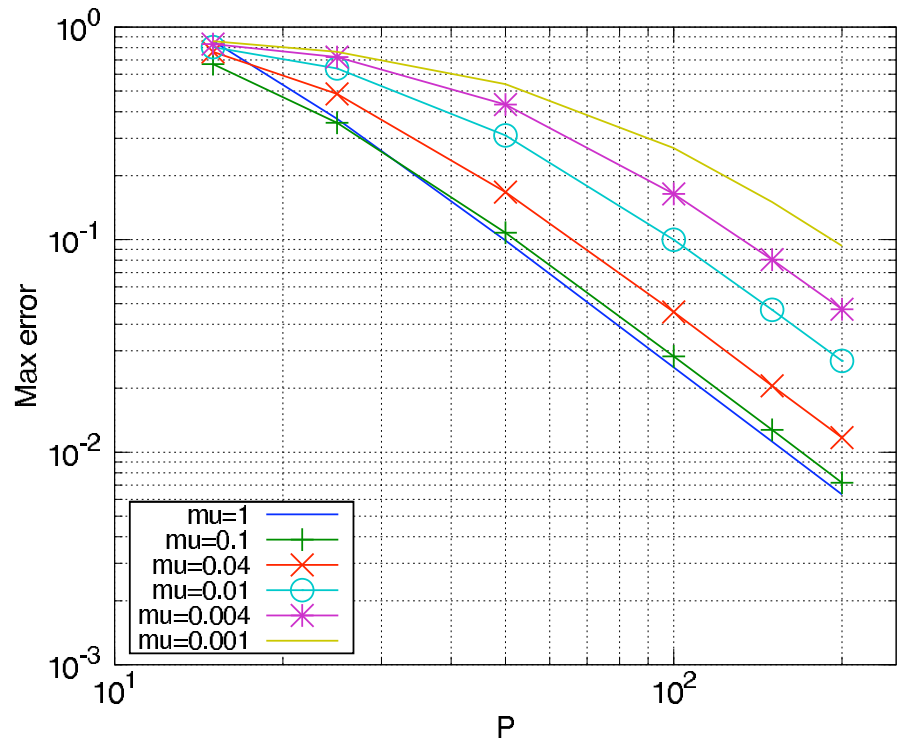

Figure 2: Max error, normalized by the max norm of the exact solution, at time $t=20$ in the numerical solution of the Rayleigh surface wave problem when $\lambda=1$ and different values of $\mu$. The error is shown as function of the number of grid points per wave length, $P=1 / h$.

tends to $\sqrt{\lambda}=1$. Hence, the temporal resolution of the surface wave only improves as $\mu \rightarrow 0$.

In this paper we use a normal mode analysis to explain the loss of accuracy as $\mu \rightarrow 0$, which corresponds to the incompressible limit of an elastic material. The normal mode analysis allows us to estimate the solution in terms of the boundary data, and makes the dependence on the material parameters transparent. We show that the solution is strongly boundary stable, except in the vicinity of the generalized eigenvalues corresponding to surface waves. Here the solution is as smooth as the boundary data, i.e., only boundary stable (see [5] for definitions of these stability concepts). We develop a modified equation model of the truncation errors in the numerical calculation, where we view the discretized boundary conditions as a perturbation of the exact boundary conditions. This analysis reveals how perturbations of the boundary conditions influence the solution, and how the material parameters enter in the relation.

To analyze the solution of (11)-(3), we follow the technique used by Kreiss, Ortiz and Petersson [5] and split the problem into two parts. First we consider a Cauchy problem, where the definition of the forcing and the initial data are extended to the whole of $\mathbb{R}^{2}(x)$. Secondly, we subtract this solution from the solution of the half-plane problem to obtain a new half-plane problem, where only the boundary data do not vanish. This is a very natural procedure because all the difficulties and many physical phenomena arise at the 
boundary. The new half-plane problem is analyzed in detail using the Fourier-Laplace transform method, leading to estimates of the solution in terms of the boundary data.

The remainder of the paper is organized in the following way. The properties of the Cauchy problem are briefly discussed in Section 2, The normal mode analysis of the half-plane problem is developed in Section 3. We discuss the eigenvalue problem in Section 3.1] 3.2, leading to necessary conditions for a well-posed problem. Boundary estimates are derived in Section 3.3. In Section 4, we use the normal mode theory to perform a modified equation analysis of the discretized boundary conditions. This analysis shows how the number of grid points per wave length must be increased to maintain a given error level in the numerical solution when $\mu \rightarrow 0$. For the second order method, the grid size must be proportional to $\mu^{1 / 2}$, while it suffices to take $h \sim \mu^{1 / 4}$ for the fourth order method. These scalings are confirmed by the numerical experiments in Section 5 , illustrating that the fourth order method is significantly more efficient than the second order approach, in particular for small values of $\mu$. Conclusions are given in Section 6 .

\section{The Cauchy problem}

In this section we consider the Cauchy problem for (1)-(2). The definitions of the forcing functions and the initial data can be smoothly extended to the whole of $\mathbb{R}^{2}(x)$. For simplicity we use the same symbols for the extended functions as for the original ones.

We start by deriving an equation for the divergence of the displacement, $\delta=u_{x}+v_{y}$, by forming the divergence of (1). This gives

$$
\delta_{t t}=(\lambda+2 \mu) \Delta \delta+G(x, y, t), \quad-\infty<(x, y)<\infty, t \geq 0,
$$

where the forcing is $G=\partial F_{1} / \partial x+\partial F_{2} / \partial y$. The divergence, $\delta=\delta(x, y, t)$, is subject to initial conditions

$$
\delta(x, y, 0)=\frac{\partial f_{10}}{\partial x}+\frac{\partial f_{11}}{\partial y}, \quad \delta_{t}(x, y, 0)=\frac{\partial f_{20}}{\partial x}+\frac{\partial f_{21}}{\partial y}, \quad-\infty<(x, y)<\infty .
$$

By first solving the wave equation for the divergence, we can (in principle) treat the divergence as a forcing in the Cauchy problems for $u$ and $v$,

$$
\left\{\begin{array}{l}
u_{t t}=\mu \Delta u+\widetilde{F}_{1}(x, y, t), \quad-\infty<(x, y)<\infty, t \geq 0, \\
v_{t t}=\mu \Delta v+\widetilde{F}_{2}(x, y, t),
\end{array}\right.
$$

where

$$
\widetilde{F}_{1}(x, y, t)=(\lambda+\mu) \delta_{x}+F_{1}(x, y, t), \quad \widetilde{F}_{2}(x, y, t)=(\lambda+\mu) \delta_{y}+F_{2}(x, y, t) .
$$

Since $\delta, u$, and $v$ all satisfy scalar wave equations, we conclude that the Cauchy problem for the elastic wave equation is well-posed. 
Note that the wave propagation speed in the wave equation for the divergence is $\sqrt{\lambda+2 \mu}$. For $G=0$, (6) admits plane wave solutions of the type

$$
\delta(x, y, t)=e^{i \omega\left(x \pm c_{p} t\right)}, \quad c_{p}=\sqrt{\lambda+2 \mu} .
$$

Hence, a wave with angular frequency $\xi=\omega c_{p}$ has wave length

$$
L_{p}=\frac{2 \pi}{\omega}=\frac{2 \pi c_{p}}{\xi}=\frac{2 \pi \sqrt{\lambda+2 \mu}}{\xi} .
$$

Note that $L_{p}$ stays bounded for $\lambda=$ const., $\mu \rightarrow 0$. By taking the curl of (11), we can also derive a scalar wave equation for the curl of the displacement, where the wave propagation speed is $\sqrt{\mu}$. Hence, the elastic wave equation also admits plane waves with wave length

$$
L_{s}=\frac{2 \pi \sqrt{\mu}}{\xi} .
$$

The length of these waves tend to zero as $\mu \rightarrow 0$.

\section{The half-plane problem}

We are interested in solutions with bounded $L^{2}$-norm and therefore we assume

$$
\int_{-\infty}^{\infty} \int_{0}^{\infty}\left(|u|^{2}+|v|^{2}\right) d x d y=\|\mathbf{u}\|^{2}<\infty, \quad \text { for every fixed } t .
$$

Throughout the remainder of the paper, $s=\eta+i \xi$ denotes a complex number where $\eta, \xi$ are real numbers. As a preliminary, we define the branch cut of $\sqrt{a+i b}$ by

$$
-\pi<\arg (a+i b) \leq \pi, \quad \arg \sqrt{a+i b}=\frac{1}{2} \arg (a+i b),
$$

where $a$ and $b$ are real numbers,

\subsection{A necessary condition for well-posedness, the eigenvalue problem}

We start with a test to find a necessary condition such that the half-plane problem is well posed.

Lemma 1. Let $F_{1}=F_{2}=0$ and $g_{1}=g_{2}=0$. The problem (11)-(3) is not well-posed if we can find a non-trivial simple wave solution of the type

$$
\begin{gathered}
u=U(x) e^{s t+i \omega y}, \quad v=V(x) e^{s t+i \omega y}, \\
|U|_{\infty}<\infty, \quad|V|_{\infty}<\infty, \quad \operatorname{Re}(s)>0, \omega \text { real. }
\end{gathered}
$$


Proof. If we have found such a solution, then

$$
u_{1}=U(\alpha x) e^{s \alpha t+i \omega \alpha y}, \quad v_{1}=V(\alpha x) e^{s \alpha t+i \omega \alpha y},
$$

is also a solution for any $\alpha>0$. Since $\operatorname{Re}(s)>0$, we can find solutions that grow arbitrarily fast in time. The problem is therefore not well-posed.

We shall now discuss whether there are such solutions. Introducing (11) into (1) gives

$$
\left\{\begin{array}{l}
\left(s^{2}+\mu \omega^{2}\right) U-(2 \mu+\lambda) U_{x x}-i(\lambda+\mu) \omega V_{x}=0, \\
\left(s^{2}+(2 \mu+\lambda) \omega^{2}\right) V-\mu V_{x x}-i(\lambda+\mu) \omega U_{x}=0,
\end{array} \quad x \geq 0 .\right.
$$

To derive boundary conditions for $U$ and $V$, we insert (11) into (3),

$$
\left\{\begin{aligned}
U_{x}+i \gamma^{2} \omega V & =0, \\
i \omega U+V_{x} & =0
\end{aligned}\right.
$$

Equation (12) is a system of linear ordinary differential equations with constant coefficients. It can be solved using the ansatz

$$
U(x)=u_{0} e^{-\kappa x}, \quad V(x)=v_{0} e^{-\kappa x} .
$$

Inserting (14) into (12) gives a linear system for $\left(u_{0}, v_{0}\right)^{T}$, which can be written

$$
\begin{aligned}
& \left(s^{2}+\mu \omega^{2}-(2 \mu+\lambda) \kappa^{2}\right) u_{0}+i(\lambda+\mu) \omega \kappa v_{0}=0, \\
& i(\lambda+\mu) \omega \kappa u_{0}+\left(s^{2}+(2 \mu+\lambda) \omega^{2}-\mu \kappa^{2}\right) v_{0}=0 .
\end{aligned}
$$

Let

$$
\zeta=s^{2}+\mu \omega^{2}-\mu \kappa^{2}
$$

Then we can write (15)-(16) as

$$
\begin{aligned}
& \left(\zeta-(\lambda+\mu) \kappa^{2}\right) u_{0}+i(\lambda+\mu) \omega \kappa v_{0}=0, \\
& i(\lambda+\mu) \omega \kappa u_{0}+\left(\zeta+(\lambda+\mu) \omega^{2}\right) v_{0}=0 .
\end{aligned}
$$

This system has a non-trivial solution if and only if its determinant is zero,

$$
\left[\zeta-(\lambda+\mu) \kappa^{2}\right]\left[\zeta+(\lambda+\mu) \omega^{2}\right]+(\lambda+\mu)^{2} \omega^{2} \kappa^{2}=0
$$

There are two possibilities. Either $\zeta=0$, or $\zeta+(\lambda+\mu)\left(\omega^{2}-\kappa^{2}\right)=0$, corresponding to

$$
\kappa= \pm \sqrt{\omega^{2}+\frac{s^{2}}{\mu}}, \quad \text { or, } \quad \kappa= \pm \sqrt{\omega^{2}+\frac{s^{2}}{(2 \mu+\lambda)}} .
$$


In appendix $\mathrm{A}$ we shall prove that there is a constant $\delta>0$ such that

$$
\operatorname{Re}\left(\sqrt{\omega^{2}+\frac{s^{2}}{\mu}}\right) \geq \delta \operatorname{Re}(s), \quad \operatorname{Re}\left(\sqrt{\omega^{2}+\frac{s^{2}}{\lambda+2 \mu}}\right) \geq \delta \operatorname{Re}(s), \quad \operatorname{Re}(s)>0 .
$$

Thus, for $\operatorname{Re}(s)>0$, there are two solutions that have bounded $L^{2}$-norm:

$$
\left(\begin{array}{l}
U(x) \\
V(x)
\end{array}\right)=e^{-\kappa_{1} x} \mathbf{u}_{1}+e^{-\kappa_{2} x} \mathbf{u}_{2}, \quad \mathbf{u}_{j}=\left(\begin{array}{c}
u_{0 j} \\
v_{0 j}
\end{array}\right), \quad j=1,2,
$$

with

$$
\kappa_{1}=\sqrt{\omega^{2}+\frac{s^{2}}{\mu}}, \quad \kappa_{2}=\sqrt{\omega^{2}+\frac{s^{2}}{\lambda+2 \mu}} .
$$

It is convenient to calculate the eigenvectors by inserting $\left(\kappa_{1}, \mathbf{u}_{1}\right)$ into (18) and $\left(\kappa_{2}, \mathbf{u}_{2}\right)$ into (17),

$$
\begin{aligned}
i(\lambda+\mu) \omega \kappa_{1} u_{01}+(\lambda+\mu) \omega^{2} v_{01} & =0 \\
-(\lambda+\mu) \omega^{2} u_{02}+i(\lambda+\mu) \omega \kappa_{2} v_{02} & =0 .
\end{aligned}
$$

Therefore,

$$
v_{01}=-\frac{i \kappa_{1}}{\omega} u_{01}, \quad v_{02}=-\frac{i \omega}{\kappa_{2}} u_{02}
$$

We summarize these results in the following lemma.

Lemma 2. Assume $\operatorname{Re}(s)>0$ and $\omega \neq 0$. Then $\kappa_{1} \neq \kappa_{2}$ and the general solution of the ordinary differential equation (12) can be written as

$$
\left(\begin{array}{c}
U(x) \\
V(x)
\end{array}\right)=u_{01}\left(\begin{array}{c}
1 \\
-\frac{i \kappa_{1}}{\omega}
\end{array}\right) e^{-\kappa_{1} x}+u_{02}\left(\begin{array}{c}
1 \\
-\frac{i \omega}{\kappa_{2}}
\end{array}\right) e^{-\kappa_{2} x}
$$

where $\kappa_{1}$ and $\kappa_{2}$ are given by (20).

Remark 1. Inserting (21) into (11) shows that all simple wave solutions satisfy

$$
\begin{aligned}
& v_{x}-u_{y}=\frac{i s^{2}}{\mu \omega} u_{01} e^{s t+i \omega y-\kappa_{1} x}, \\
& u_{x}+v_{y}=-\frac{s^{2}}{(\lambda+2 \mu) \kappa_{2}} u_{02} e^{s t+i \omega y-\kappa_{2} x} .
\end{aligned}
$$

Hence, $u_{01}$ and $u_{02}$ are proportional to the curl and divergence of the solution, respectively. 
Introducing (21) into the boundary conditions (13) gives

$$
\begin{aligned}
\left(1-\gamma^{2}\right) \kappa_{1} \kappa_{2} u_{01}+\left(\kappa_{2}^{2}-\gamma^{2} \omega^{2}\right) u_{02} & =0 \\
\left(\kappa_{1}^{2}+\omega^{2}\right) u_{01}+2 \omega^{2} u_{02} & =0 .
\end{aligned}
$$

The linear system (24)-(25) has a non-trivial solution if and only if its determinant is zero,

$$
\Delta=: 2 \omega^{2}\left(1-\gamma^{2}\right) \kappa_{1} \kappa_{2}-\left(\kappa_{2}^{2}-\gamma^{2} \omega^{2}\right)\left(\kappa_{1}^{2}+\omega^{2}\right)=0 .
$$

Since $1-\gamma^{2}=2 \mu /(\lambda+2 \mu)$, we can write (26) in the form

$$
\mu(\lambda+2 \mu) \Delta=4 \mu^{2} \omega^{4}\left(\sqrt{1+\frac{s^{2}}{\omega^{2} \mu}} \sqrt{1+\frac{s^{2}}{\omega^{2}(\lambda+2 \mu)}}-\left(1+\frac{s^{2}}{2 \mu \omega^{2}}\right)^{2}\right)=: 4 \mu^{2} \omega^{4} \varphi(\tilde{s}),
$$

where

$$
\varphi(\tilde{s})=: \sqrt{1+\tilde{s}^{2}} \sqrt{1+\frac{\mu \tilde{s}^{2}}{\lambda+2 \mu}}-\left(1+\frac{\tilde{s}^{2}}{2}\right)^{2}, \quad \tilde{s}=\frac{s}{|\omega| \sqrt{\mu}} .
$$

Note that the zeros of the determinant (26) are the solutions of $\varphi(\tilde{s})=0$.

Lemma 3. Assume $\omega \neq 0$. The function $\varphi(\tilde{s})$ does not have any zeros for $\operatorname{Re}(\tilde{s})>0$.

Proof. Assume there was a solution of $\varphi(\tilde{s})=0$ with $\operatorname{Re}(\tilde{s})>0$. It would correspond to a non-trivial solution $\left(u_{01}, u_{02}\right)$ of (24)-(25). There would therefore be a simple wave solution (11) where $U(x)$ and $V(x)$ are given by (21). This simple wave solution would have $\operatorname{Re}(s)=|\omega| \sqrt{\mu} \operatorname{Re}(\tilde{s})>0$, and for this reason, its elastic energy (44) would grow exponentially in time. However, this is contradicted by the energy estimate (5), which says that the elastic energy must be constant in time. There can therefore be no simple wave solutions for $\operatorname{Re}(s)>0$, and the function $\varphi(\tilde{s})$ can not have any zeros for $\operatorname{Re}(\tilde{s})>0$.

As a consequence of this lemma,

Theorem 1. The elastic wave equation (1)-(3) with $F_{1}=F_{2}=0$ and $g_{1}=g_{2}=0$, has no simple wave solutions of the type (11), other than the trivial solution $u=v=0$.

Because (12)-(13) define an eigenvalue problem, we can also phrase the theorem as

Theorem 2. The eigenvalue problem (12)-(13) has no eigenvalues with $\operatorname{Re}(s)>0$.

\subsection{Generalized eigenvalues}

We shall now calculate the generalized eigenvalues, i.e., roots of the determinant (27) in the limit $\operatorname{Re}(\tilde{s}) \rightarrow 0+$. We need to discuss $\tilde{s}=i \tilde{\xi}, \tilde{\xi}$ real, and the zeros are given by

$$
\varphi(i \tilde{\xi})=: \sqrt{1-\tilde{\xi}^{2}} \cdot \sqrt{1-\frac{\mu \tilde{\xi}^{2}}{2 \mu+\lambda}}-\left(1-\frac{\tilde{\xi}^{2}}{2}\right)^{2}=0, \quad \tilde{s}^{2}=-\tilde{\xi}^{2} .
$$

We have 
Lemma 4. Equation (28) has the solution $\tilde{\xi}=0$, and exactly two solutions $\tilde{s}_{0}= \pm i \tilde{\xi}_{0}$ with $0<\tilde{\xi}_{0}<1$. There are no solutions with $\tilde{\xi}^{2} \geq 1$.

Proof. Inserting $\tilde{\xi}=0$ into (28) shows that it is a solution. Clearly, there are no solutions for $1 \leq \tilde{\xi}^{2}<(2 \mu+\lambda) / \mu$ because the first square root is purely imaginary and the second square root is real. Also, the second term in $\varphi$ is always real and negative. For $\tilde{\xi}^{2} \geq(2 \mu+\lambda) / \mu$, both square roots are purely imaginary and their product is real and negative. Hence both terms in $\varphi$ are real and negative. We conclude that there are no solutions for $\tilde{\xi}^{2} \geq 1$.

To analyze $0<\tilde{\xi}^{2}<1$, we denote $\tilde{\lambda}=\lambda / \mu$ and observe that the function

$$
\psi(\sigma)=(1-\sigma)\left(1-\frac{\sigma}{2+\tilde{\lambda}}\right)-\left(1-\frac{\sigma}{2}\right)^{4}, \quad \sigma=\tilde{\xi}^{2},
$$

has the same roots as $\varphi$. It has the properties

1.

$$
\psi(0)=0, \quad \psi(1)=-\frac{1}{16}<0
$$

2.

$$
d \psi / d \sigma=: \psi^{\prime}(\sigma)=-\left(1+\frac{1}{2+\tilde{\lambda}}\right)+\frac{2 \sigma}{2+\tilde{\lambda}}+2\left(1-\frac{\sigma}{2}\right)^{3},
$$

that is,

$$
\psi^{\prime}(0)=\frac{1+\tilde{\lambda}}{2+\tilde{\lambda}}>0, \quad \psi^{\prime}(1)=-\frac{2+3 \tilde{\lambda}}{8+4 \tilde{\lambda}}<0 .
$$

3.

$$
\psi^{\prime \prime}(\sigma)=\frac{2}{2+\tilde{\lambda}}-3\left(1-\frac{\sigma}{2}\right)^{2}
$$

that is,

$$
\psi^{\prime \prime}(0)=-\frac{4+3 \tilde{\lambda}}{2+\tilde{\lambda}}<0, \quad \psi^{\prime \prime}(\sigma)=0 \text { for } \frac{\sigma}{2}=1 \pm \sqrt{\frac{2}{3(2+\tilde{\lambda})}} .
$$

Thus $\psi^{\prime \prime}(\sigma)$ has at most one sign change in $0 \leq \sigma \leq 1$. Properties $1-3$ show that $\psi^{\prime}$ has one sign change and the lemma follows.

In Table 1 we have calculated the scaled generalized eigenvalues $\tilde{s}_{0}^{2}=-\tilde{\xi}_{0}^{2}$ for some values of $\lambda / \mu$. Note that all values remain bounded in the limit $\lambda / \mu \rightarrow \infty$, i.e. $\mu \rightarrow 0$ when $\lambda=$ const.

Differentiating (27) gives

$$
\varphi^{\prime}(\tilde{s})=\frac{\tilde{s}}{\sqrt{1+\tilde{s}^{2}}} \sqrt{1+\frac{\mu \tilde{s}^{2}}{2 \mu+\lambda}}+\frac{\tilde{s} \mu}{2 \mu+\lambda} \frac{\sqrt{1+\tilde{s}^{2}}}{\sqrt{1+\frac{\mu \tilde{s}^{2}}{2 \mu+\lambda}}}-2 \tilde{s}\left(1+\frac{\tilde{s}^{2}}{2}\right) .
$$




\begin{tabular}{|c|c|c|c|c|}
\hline$\lambda / \mu$ & $\tilde{s}_{0}^{2}=-\tilde{\xi}_{0}^{2}$ & $\kappa_{10} /|\omega|$ & $\kappa_{20} /|\omega|$ & $\left|\varphi^{\prime}\left(\tilde{s}_{0}\right)\right|$ \\
\hline \hline 0 & -0.7639 & 0.4858 & 0.7861 & 0.6036 \\
1 & -0.8452 & 0.3933 & 0.8474 & 1.0610 \\
4 & -0.8877 & 0.3350 & 0.9230 & 1.6045 \\
8 & -0.8991 & 0.3175 & 0.9539 & 1.8360 \\
$\infty$ & -0.9126 & 0.2955 & 1 & 2.1936 \\
\hline
\end{tabular}

Table 1: Coefficients in the solution at the generalized eigenvalues $s_{0}= \pm i \sqrt{\mu}|\omega| \tilde{\xi}_{0}$, for some values of $\lambda / \mu$.

Because $\varphi\left(\tilde{s}_{0}\right)=0$, (27) gives

$$
\sqrt{1+\tilde{s}_{0}^{2}} \sqrt{1+\frac{\mu \tilde{s}_{0}^{2}}{2 \mu+\lambda}} \frac{\varphi^{\prime}\left(\tilde{s}_{0}\right)}{\tilde{s}_{0}}=1+\frac{\mu \tilde{s}_{0}^{2}}{2 \mu+\lambda}+\frac{\mu\left(1+\tilde{s}_{0}^{2}\right)}{2 \mu+\lambda}-2\left(1+\frac{\tilde{s}_{0}^{2}}{2}\right)^{3}:=C_{0},
$$

where $C_{0}=C_{0}(\lambda / \mu)$ is real. Since $\tilde{s}_{0}$ is purely imaginary, $\varphi^{\prime}\left(\tilde{s}_{0}\right)$ is also purely imaginary. We report numerical values of $\left|\varphi^{\prime}\left(\tilde{s}_{0}\right)\right|$ in Table 1, demonstrating that $\varphi^{\prime}\left(\tilde{s}_{0}\right)$ is bounded away from zero for all values of $\lambda / \mu \geq 0$. Therefore, $\varphi(\tilde{s})$ has a first order zero at the generalized eigenvalues $\tilde{s}_{0}= \pm i \tilde{\xi}_{0}$.

To calculate the eigenfunctions corresponding to the generalized eigenvalues $s_{0}= \pm i \xi_{0}$, we consider the two boundary conditions (13). Evaluating the general solution (21) gives

$$
i \omega U+V_{x}=i \omega\left(\left(1+\frac{\kappa_{1}^{2}}{\omega^{2}}\right) u_{01}+2 u_{02}\right), \quad x=0 .
$$

At the generalized eigenvalues,

$$
\kappa_{1}^{2}=\omega^{2}\left(1+\tilde{s}_{0}^{2}\right)=\omega^{2}\left(1-\tilde{\xi}_{0}^{2}\right) .
$$

Hence, $i \omega U+V_{x}=0$ if

$$
\left(2-\tilde{\xi}_{0}^{2}\right) u_{01}+2 u_{02}=0 .
$$

If relation (31) is satisfied, also $U_{x}+i \gamma^{2} \omega V=0$. The eigenfunction corresponding to $s_{0}= \pm i \xi_{0}$ is therefore given by

$$
\left(\begin{array}{l}
u \\
v
\end{array}\right)=e^{ \pm i \xi_{0} t+i \omega y-\kappa_{10} x}\left(\begin{array}{c}
1 \\
-\frac{i \kappa_{10}}{\omega}
\end{array}\right)+\frac{1}{2}\left(\tilde{\xi}_{0}^{2}-2\right) e^{ \pm i \xi_{0} t+i \omega y-\kappa_{20} x}\left(\begin{array}{c}
1 \\
-\frac{i \omega}{\kappa_{20}}
\end{array}\right),
$$


where

$$
\kappa_{10}=|\omega| \sqrt{1-\tilde{\xi}_{0}^{2}}, \quad \kappa_{20}=|\omega| \sqrt{1-\frac{\mu \tilde{\xi}_{0}^{2}}{\lambda+2 \mu}}, \quad \tilde{\xi}_{0}=\frac{\xi_{0}}{|\omega| \sqrt{\mu}} .
$$

These eigenfunctions, also known as Rayleigh waves (see e.g. Achenbach [1], §5.11), represent surface waves that propagate in the positive or negative $y$-direction.

Now we consider the potential generalized eigenvalue $\tilde{s}=0$. Relations (27) and (29) show that both $\varphi=0$ and $\partial \varphi / \partial \tilde{s}=0$ for $\tilde{s}=0$. Differentiating (29) shows that $\partial^{2} \varphi / \partial \tilde{s}^{2} \neq 0$ for $\tilde{s}=0$. Thus $\varphi(\tilde{s})$ has a zero of order two at $\tilde{s}=0$. However, for $\tilde{s} \rightarrow 0$, (20) show that both $\kappa_{1} \rightarrow|\omega|$ and $\kappa_{2} \rightarrow|\omega|$. In this limit, boundary conditions (24) and (25) give

$$
u_{01}+u_{02}=0, \quad \tilde{s} \rightarrow 0
$$

Expanding the general solution (21) around $\tilde{s}=0$ shows that the eigenfunction vanishes identically in this limit. Thus $\tilde{s}=0$ is not a generalized eigenvalue.

\subsection{Boundary forcing}

As we discussed in the introduction, we split the solution of the half-plane problem (11)-(3) into a Cauchy problem and a new half-plane problem, where only the boundary data do not vanish. Hence, the Cauchy problem satisfies the initial conditions and the interior forcing function. Its solution drives the solution of the new half-plane problem through a modified boundary forcing function. For example, when the half-plane problem (11)-(3) has an interior forcing function with compact support in $\bar{\Omega}$, the solution of the Cauchy problem consists of waves propagating outwards from $\bar{\Omega}$. The gradient of these waves along $x=0$ enter in the boundary forcing functions for the new half-plane problem.

The estimates obtained in this and the following sections are expressed in FourierLaplace transformed space. It is clear that all these estimates have their counterpart in physical space. To understand the relation between both types of estimates, we refer to chapter 7.4 of [3] or chapter 10 of [2].

We consider (11)-(3) with homogeneous initial data and internal forcing, $F_{1}=F_{2}=0$. We Laplace transform the problem with respect to $t$, Fourier transform it with respect to $y$, and denote the dual variables by $s$ and $\omega$, respectively. Here $\omega$ is a real number and $s$ is complex. We obtain,

$$
\left\{\begin{array}{l}
\left(s^{2}+\mu \omega^{2}\right) \hat{u}-(2 \mu+\lambda) \hat{u}_{x x}-i \omega(\lambda+\mu) \hat{v}_{x}=0, \\
\left(s^{2}+(2 \mu+\lambda) \omega^{2}\right) \hat{v}-\mu \hat{v}_{x x}-i \omega(\lambda+\mu) \hat{u}_{x}=0
\end{array} \quad x \geq 0, \operatorname{Re}(s)>0,\right.
$$

subject to the boundary condition

$$
\left\{\begin{array}{l}
\hat{u}_{x}+i \gamma^{2} \omega \hat{v}=\hat{g}_{1}(\omega, s), \\
i \omega \hat{u}+\hat{v}_{x}=\hat{g}_{2}(\omega, s)
\end{array} \quad x=0 .\right.
$$


Note that $(\hat{u}, \hat{v})^{T}$ satisfy the same differential equation as $(U, V)^{T}$ in (12). By Lemma2, the general solution is of the form (21), i.e.,

$$
\left(\begin{array}{l}
\hat{u}(x) \\
\hat{v}(x)
\end{array}\right)=\hat{u}_{01}\left(\begin{array}{c}
1 \\
-\frac{i \kappa_{1}}{\omega}
\end{array}\right) e^{-\kappa_{1} x}+\hat{u}_{02}\left(\begin{array}{c}
1 \\
-\frac{i \omega}{\kappa_{2}}
\end{array}\right) e^{-\kappa_{2} x} .
$$

In the following, we assume $\omega \neq 0$. The case $\omega \rightarrow 0$ will be studied separately in appendix B.

By inserting (35) into boundary condition (34), we get

$$
\begin{aligned}
\left(1-\gamma^{2}\right) \kappa_{1} \kappa_{2} \hat{u}_{01}+\left(\kappa_{2}^{2}-\gamma^{2} \omega^{2}\right) \hat{u}_{02} & =-\kappa_{2} \hat{g}_{1}, \\
\left(\kappa_{1}^{2}+\omega^{2}\right) \hat{u}_{01}+2 \omega^{2} \hat{u}_{02} & =-i \omega \hat{g}_{2} .
\end{aligned}
$$

This system corresponds to (24)-(25) with an inhomogeneous right hand side. In terms of the scaled variable $\tilde{s}$ defined by (27),

$$
\kappa_{1}=|\omega| \sqrt{1+\tilde{s}^{2}}, \quad \kappa_{2}=|\omega| \sqrt{1+\frac{\mu \tilde{s}^{2}}{\lambda+2 \mu}} .
$$

After some algebra, the system for $\left(\hat{u}_{01}, \hat{u}_{02}\right)^{T}$ becomes

$$
\begin{gathered}
\sqrt{1+\tilde{s}^{2}} \sqrt{1+\frac{\tilde{s}^{2} \mu}{2 \mu+\lambda}} \hat{u}_{01}+\left(1+\frac{\tilde{s}^{2}}{2}\right) \hat{u}_{02}=-\frac{(\lambda+2 \mu) \hat{g}_{1}}{2 \mu|\omega|} \sqrt{1+\frac{\tilde{s}^{2} \mu}{2 \mu+\lambda}} \\
\left(1+\frac{\tilde{s}^{2}}{2}\right) \hat{u}_{01}+\hat{u}_{02}=-\frac{i \hat{g}_{2}}{2 \omega} .
\end{gathered}
$$

The determinant of (37))-(38) is

$$
\varphi(\tilde{s})=\sqrt{1+\tilde{s}^{2}} \sqrt{1+\frac{\tilde{s}^{2} \mu}{2 \mu+\lambda}}-\left(1+\frac{\tilde{s}^{2}}{2}\right)^{2},
$$

where the function $\varphi(\tilde{s})$ was previously defined by (27). To solve the system, we eliminate $\hat{u}_{02}$ from (38) and insert in (37),

$$
\varphi(\tilde{s}) \hat{u}_{01}=-\frac{(\lambda+2 \mu) \hat{g}_{1}}{2 \mu|\omega|} \sqrt{1+\frac{\tilde{s}^{2} \mu}{2 \mu+\lambda}}+\frac{i \hat{g}_{2}}{2 \omega}\left(1+\frac{\tilde{s}^{2}}{2}\right) .
$$

Inserting this expression into (38) gives

$$
\varphi(\tilde{s}) \hat{u}_{02}=\frac{(\lambda+2 \mu) \hat{g}_{1}}{2 \mu|\omega|} \sqrt{1+\frac{\mu \tilde{s}^{2}}{\lambda+2 \mu}}\left(1+\frac{\tilde{s}^{2}}{2}\right)-\frac{i \hat{g}_{2}}{2 \omega} \sqrt{1+\tilde{s}^{2}} \sqrt{1+\frac{\mu \tilde{s}^{2}}{\lambda+2 \mu}} .
$$


Hence, the system (37)-(38) becomes singular exactly at the roots of $\varphi(\tilde{s})=0$. For $\operatorname{Re}(s) \geq 0$, Lemmas 3 and 4 prove that this can only happen at the generalized eigenvalues. The general theory of [5] tells us that, away from the generalized eigenvalues, $|\varphi(\tilde{s})|^{-1}$ is bounded and the problem is therefore strongly boundary stable.

We want to estimate the solution on the boundary in terms of the boundary forcing. For $x=0$, the general solution (35) satisfies

$$
\left\{\begin{array}{l}
\hat{u}(0)=\hat{u}_{01}+\hat{u}_{02} \\
\hat{v}(0)=-\frac{i \kappa_{1}}{\omega} \hat{u}_{01}-\frac{i \omega}{\kappa_{2}} \hat{u}_{02}=-\frac{i|\omega|}{\omega} \sqrt{1+\tilde{s}^{2}} \hat{u}_{01}-\frac{i \omega}{|\omega|}\left(1+\frac{\mu \tilde{s}^{2}}{\lambda+2 \mu}\right)^{-1 / 2} \hat{u}_{02}
\end{array}\right.
$$

We now discuss how the solution behaves close to the generalized eigenvalues $\tilde{s}_{0}=$ $\pm i \tilde{\xi}_{0}$. By Lemma 4, we have $0<\tilde{\xi}_{0}<1$ and both $\kappa_{1}$ and $\kappa_{2}$ are real. Since $\varphi\left(\tilde{s}_{0}\right)=0$, Taylor expansion gives

$$
\varphi(\tilde{s})=\left(\tilde{s}-\tilde{s}_{0}\right) \varphi^{\prime}\left(\tilde{s}_{0}\right)+\mathcal{O}\left(\left|\tilde{s}-\tilde{s}_{0}\right|^{2}\right) .
$$

Formula (30) and Table 1 shows that $\left|\varphi^{\prime}\left(\tilde{s}_{0}\right)\right| \geq C_{0} \simeq 0.6$ for all $\lambda / \mu \geq 0$. We have $s-s_{0}=\eta+i\left(\xi-\xi_{0}\right)$, and to leading order in $0<\eta \ll 1$,

$$
\varphi(\tilde{s})=\frac{s-s_{0}}{\sqrt{\mu}|\omega|} \varphi^{\prime}\left(\tilde{s}_{0}\right)+\mathcal{O}\left(\eta^{2}\right)
$$

which leads to the estimates

$$
\left|s-s_{0}\right| \geq \eta, \quad|\varphi(\tilde{s})| \geq \frac{\eta}{\sqrt{\mu}|\omega|} C_{0}, \quad \eta>0 .
$$

For $\eta>0$, the system (37)-(38) is non-singular and we can substitute (43) into the solution formulas (39)-(40) to calculate $\hat{u}_{01}$ and $\hat{u}_{02}$. Inserting these values in (41) and applying the triangle inequality proves the following lemma.

Lemma 5. Let $s= \pm i \xi_{0}+s^{\prime}, 0<\left|s^{\prime}\right| \ll 1$, where $\xi_{0}=\sqrt{\mu}|\omega| \tilde{\xi}_{0}$ and $\varphi\left( \pm i \tilde{\xi}_{0}\right)=0$ with $0<\tilde{\xi}_{0}<1$. Also assume $\operatorname{Re}\left(s^{\prime}\right)=\eta>0$. Then, the solution of (33)-(34) satisfies the boundary estimate

$$
\begin{aligned}
& |\hat{u}(0)| \leq \frac{K}{\eta}\left[\frac{2 \mu+\lambda}{\sqrt{\mu}}\left|\hat{g}_{1}\right|+\sqrt{\mu}\left|\hat{g}_{2}\right|\right], \\
& |\hat{v}(0)| \leq \frac{K}{\eta}\left[\frac{2 \mu+\lambda}{\sqrt{\mu}}\left|\hat{g}_{1}\right|+\sqrt{\mu}\left|\hat{g}_{2}\right|\right],
\end{aligned}
$$

where the constant $K>0$ is independent of $\mu$ and $\lambda$. The solution is as smooth as the boundary data and is therefore boundary stable. The solution operator has a simple pole at $s_{0}= \pm i \xi_{0}$ and as a consequence, the solution in physical space grows linearly in time. The growth rate is proportional to $\left|\hat{g}_{1}\right| / \sqrt{\mu}$ as $\mu \rightarrow 0$. 
We shall now discuss the case $s \rightarrow 0$ in more detail. We assume $|\omega| \geq \omega_{0}>0$, which implies $\tilde{s} \rightarrow 0$. Note that the eigenvectors in the general solution (35) become linearly dependent in the limit, because both $\kappa_{1}=|\omega|$ and $\kappa_{2}=|\omega|$ for $\tilde{s}=0$. We therefore assume $\operatorname{Re} \tilde{s}=\tilde{\eta}>0$, and study the the solution in the limit $|\tilde{s}| \rightarrow 0$.

Because $|\tilde{s}| \ll 1$, we can simplify (37) to

$$
\left(1+\frac{\tilde{s}^{2}}{2}\left(1+\frac{\mu}{2 \mu+\lambda}\right)\right) \hat{u}_{01}+\left(1+\frac{\tilde{s}^{2}}{2}\right) \hat{u}_{02}=-\frac{(2 \mu+\lambda) \hat{g}_{1}}{2 \mu|\omega|}\left(1+\frac{\tilde{s}^{2}}{2} \frac{\mu}{2 \mu+\lambda}\right) .
$$

We eliminate $\hat{u}_{02}$ using (38) and obtain

$$
\begin{aligned}
\left(1+\frac{\tilde{s}^{2}}{2}\left(1+\frac{\mu}{2 \mu+\lambda}\right)-\left(1+\frac{\tilde{s}^{2}}{2}\right)^{2}\right) & \hat{u}_{01} \\
& =-\frac{(2 \mu+\lambda) \hat{g}_{1}}{2 \mu|\omega|}\left(1+\frac{\tilde{s}^{2}}{2} \frac{\mu}{2 \mu+\lambda}\right)+\frac{i \hat{g}_{2}}{2 \omega}\left(1+\frac{\tilde{s}^{2}}{2}\right) .
\end{aligned}
$$

For small $|\tilde{s}|^{2}$ we obtain to first approximation

$$
\tilde{s}^{2} \hat{u}_{01}=\frac{(\lambda+2 \mu)^{2} \hat{g}_{1}}{(\lambda+\mu) \mu|\omega|}-\frac{i(\lambda+2 \mu) \hat{g}_{2}}{(\lambda+\mu) \omega} .
$$

Relation (38) can be written

$$
\begin{aligned}
\hat{u}_{01}+\hat{u}_{02} & =-\frac{\tilde{s}^{2}}{2} \hat{u}_{01}-\frac{i \hat{g}_{2}}{2 \omega} \\
& =-\frac{(\lambda+2 \mu)^{2} \hat{g}_{1}}{2(\lambda+\mu) \mu|\omega|}+\frac{i \mu \hat{g}_{2}}{2(\lambda+\mu) \omega} .
\end{aligned}
$$

The solution on the boundary is given by (41). The first component satisfies $\hat{u}(0)=$ $\hat{u}_{01}+\hat{u}_{02}$, and (49) shows that $\hat{u}(0)$ is bounded independently of $\tilde{s}$. The expression for the second component can be simplified for $|\tilde{s}| \ll 1$. We have to leading order

$$
\begin{array}{r}
\hat{v}(0)=-\frac{i|\omega|}{\omega}\left(1+\frac{\tilde{s}^{2}}{2}\right) \hat{u}_{01}-\frac{i|\omega|}{\omega}\left(1-\frac{1}{2} \frac{\mu \tilde{s}^{2}}{\lambda+2 \mu}\right) \hat{u}_{02} \\
=-\frac{i|\omega|}{\omega}\left(\hat{u}_{01}+\hat{u}_{02}\right)-\frac{i|\omega|}{\omega} \frac{\tilde{s}^{2}}{2}\left(\hat{u}_{01}-\frac{\mu}{\lambda+2 \mu} \hat{u}_{02}\right) .
\end{array}
$$

Therefore, also $\hat{v}(0)$ is bounded independently of $\tilde{s}$. The factor $\omega$ in the denominator of the right hand side of (49) gives the desired result that our problem is strongly boundary stable at $\tilde{s}=0$. 


\section{Influence of truncation errors on the generalized eigenvalues}

Consider the homogeneous differential equations (11) with boundary conditions (3) . Let

$$
g_{1}=\alpha_{1} h^{2} u_{x x x}+\alpha_{2} h^{2} v_{y y y}, \quad g_{2}=\beta_{1} h^{2} v_{x x x}+\beta_{2} h^{2} u_{y y y},
$$

denote the principal part of the truncation error in a second order accurate method with grid size $h$. We can think of boundary conditions (3) with boundary data (51) as modified homogeneous boundary conditions. Again, we solve the problem using the technique of Section 3.3 in terms of the simple wave ansatz (35). By section 3.3, the modified boundary conditions become

$$
\begin{array}{r}
\left(1-\gamma^{2}\right) \kappa_{1} \kappa_{2} \hat{u}_{01}+\left(\kappa_{2}^{2}-\gamma^{2} \omega^{2}\right) \hat{u}_{02}+\kappa_{2} \hat{g}_{1}=0 \\
\left(\omega^{2}+\kappa_{2}^{2}\right) \hat{u}_{01}+2 \omega^{2} \hat{u}_{02}+i \omega \hat{g}_{2}=0
\end{array}
$$

where

$$
\begin{aligned}
& \hat{g}_{1}=-\alpha_{1} h^{2}\left(\kappa_{1}^{3} \hat{u}_{01}+\kappa_{2}^{3} \hat{u}_{02}\right)-\alpha_{2} h^{2}\left(\omega^{2} \kappa_{1} \hat{u}_{01}+\frac{\omega^{4}}{\kappa_{2}} \hat{u}_{02}\right), \\
& \hat{g}_{2}=i \beta_{1} h^{2}\left(\frac{\kappa_{1}^{4}}{\omega} \hat{u}_{01}+\kappa_{2}^{2} \omega \hat{u}_{02}\right)-i \beta_{2} h^{2} \omega^{3}\left(\hat{u}_{01}+\hat{u}_{02}\right) .
\end{aligned}
$$

For small $\mu$, the main effect comes from $\hat{g}_{1}$. For simplicity, we therefore assume that $\hat{g}_{2}=0$ and obtain the equations

$$
\hat{g}_{1}=-h^{2}\left(\alpha_{1} \kappa_{1}^{3}+\alpha_{2} \omega^{2} \kappa_{1}\right) \hat{u}_{01}-h^{2}\left(\alpha_{1} \kappa_{2}^{3}+\alpha_{2} \frac{\omega^{4}}{\kappa_{2}}\right) \hat{u}_{02}, \quad \hat{g}_{2}=0 .
$$

Introducing the scaled variable $\tilde{s}$ and the formulas for $\kappa_{j}$ according to (36) gives us relations (37)-(38) with $\hat{g}_{2}=0$. By using the homogeneous equation (38), we can eliminate $\hat{u}_{02}$ from (37) and (52), resulting in the solution formula (39) with $\hat{g}_{2}=0$, and

$$
\hat{g}_{1}=-h^{2}\left(\alpha_{1} \kappa_{1}^{3}+\alpha_{2} \omega^{2} \kappa_{1}\right) \hat{u}_{01}+h^{2}\left(1+\frac{\tilde{s}^{2}}{2}\right)\left(\alpha_{1} \kappa_{2}^{3}+\alpha_{2} \frac{\omega^{4}}{\kappa_{2}}\right) \hat{u}_{01} .
$$

Hence, the solution formula (39) defines a perturbed eigenvalue problem that can be written in the form

$$
\varphi(\tilde{s}) \hat{u}_{01}=\theta\left(\tilde{s}^{2}\right) \hat{u}_{01} .
$$

Since

$$
\frac{\kappa_{2}}{|\omega|}=\sqrt{1+\frac{\tilde{s}^{2} \mu}{\lambda+2 \mu}}
$$


we have

$$
\theta\left(\tilde{s}^{2}\right)=\frac{(\lambda+2 \mu) h^{2}}{2 \mu|\omega|} \frac{\kappa_{2}}{|\omega|}\left(\alpha_{1} \kappa_{1}^{3}+\alpha_{2} \omega^{2} \kappa_{1}-\left(1+\frac{\tilde{s}^{2}}{2}\right)\left(\alpha_{1} \kappa_{2}^{3}+\alpha_{2} \frac{\omega^{4}}{\kappa_{2}}\right)\right) .
$$

We assume now that that $\lambda / \mu \gg 1$. For the unperturbed problem, the properties of the generalized eigenvalues are given in Table 1,

$$
\tilde{s}_{0}^{2} \simeq-0.9, \quad \frac{\kappa_{1}}{|\omega|} \simeq 0.3, \quad \frac{\kappa_{2}}{|\omega|} \simeq 1
$$

Therefore,

$$
\begin{aligned}
\theta\left(\tilde{s}_{0}^{2}\right) & \simeq \frac{\lambda h^{2}}{2 \mu|\omega|} \frac{\kappa_{2}}{|\omega|}\left(\alpha_{1} \frac{\kappa_{1}^{3}}{|\omega|^{3}}|\omega|^{3}+\alpha_{2} \omega^{2} \frac{\kappa_{1}}{|\omega|}|\omega|-0.55\left(\alpha_{1} \frac{\kappa_{2}^{3}}{|\omega|^{3}}|\omega|^{3}+\alpha_{2} \omega^{2}|\omega| \frac{|\omega|}{\kappa_{2}}\right)\right) \\
& \simeq \frac{\lambda h^{2} \omega^{2}}{2 \mu}\left(0.027 \alpha_{1}+0.3 \alpha_{2}-0.55\left(\alpha_{1}+\alpha_{2}\right)\right) .
\end{aligned}
$$

We want to estimate how sensitive the generalized eigenvalues $s_{0}= \pm i \xi_{0}$ are to truncation error perturbations. We perturb $\varphi(\tilde{s})$ around $\tilde{s}_{0}$. For $\lambda / \mu \gg 1$, Table 1 and (30) gives

$$
\begin{aligned}
\frac{\kappa_{1}}{|\omega|} \frac{\kappa_{2}}{|\omega|} \frac{\varphi^{\prime}\left(\tilde{s}_{0}\right)}{\tilde{s}_{0}} & \simeq \frac{\kappa_{2}^{2}}{\omega^{2}}-2\left(1+\frac{\tilde{s}_{0}^{2}}{2}\right)^{3} \simeq 0.67 \\
\varphi^{\prime}\left(\tilde{s}_{0}\right) & \simeq \tilde{s}_{0} \frac{0.67}{0.3} \simeq \pm 2.12 i
\end{aligned}
$$

The Taylor expansion (42) gives for small $h \omega$,

$$
\left(\tilde{s}-\tilde{s}_{0}\right) \varphi^{\prime}\left(\tilde{s}_{0}\right)=\theta\left(\tilde{s}_{0}\right)
$$

We get

$$
\tilde{s}-\tilde{s}_{0} \simeq \frac{\theta\left(\tilde{s}_{0}^{2}\right)}{\varphi^{\prime}\left(\tilde{s}_{0}\right)} \simeq \mp \frac{\lambda h^{2} \omega^{2}}{2 \mu}\left(0.027 \alpha_{1}+0.3 \alpha_{2}-0.55\left(\alpha_{1}+\alpha_{2}\right)\right) \frac{i}{2.12} .
$$

We now make some observations. Because $\theta\left(\tilde{s}_{0}^{2}\right)$ is real, the generalized eigenvalue is perturbed along the imaginary axis and remains purely imaginary. Hence the perturbed problem is well-posed. The value of the perturbed generalized eigenvalue determines the phase velocity of surface waves in the numerical solution. To avoid large phase errors, we must therefore keep the perturbation of the generalized eigenvalue small. If we accept a relative error in the phase speed of size $\epsilon$, where $0<\epsilon \ll 1$, we have to choose the grid size $h$ such that

$$
\frac{\lambda h^{2} \omega^{2}\left|\alpha_{0}\right|}{\mu}=\epsilon<<1
$$


If the computational grid has $P$ grid points per wave length $L=2 \pi /|\omega|$, we get

$$
h=\frac{L}{P}=\frac{2 \pi}{|\omega| P}, \quad h|\omega|=\frac{2 \pi}{P}, \quad P=2 \pi\left(\frac{\left|\alpha_{0}\right|}{\epsilon} \frac{\lambda}{\mu}\right)^{1 / 2} .
$$

Hence, the number of grid points per wave length must be proportional to $\sqrt{\lambda / \mu}$ to maintain the accuracy as $\mu / \lambda \rightarrow 0$.

For a fourth order accurate method, where the leading order truncation error terms are

$$
g_{1}=\alpha_{1}^{\prime} h^{4} \frac{\partial^{5} u}{\partial x^{5}}+\alpha_{2}^{\prime} h^{4} \frac{\partial^{5} v}{\partial y^{5}}
$$

equation (54) is replaced by

$$
\frac{\lambda h^{4} \omega^{4}\left|\alpha_{0}^{\prime}\right|}{\mu}=\epsilon<<1
$$

The number of grid points per wave length to maintain an $\epsilon$-error in the phase velocity now becomes

$$
P=2 \pi\left(\frac{\left|\alpha_{0}^{\prime}\right| \lambda}{\epsilon \mu}\right)^{1 / 4}
$$

Therefore, as $\mu / \lambda \rightarrow 0$, the number of grid points per wave length grows much slower for the 4 th than the 2nd order accurate method.

For other truncation error perturbations of the boundary conditions, such as a $u_{x x x x}$ term in $g_{1}, \theta\left(\tilde{s}_{0}^{2}\right)$ becomes complex. If the truncation error coefficient has the wrong sign, the perturbed problem gets eigenvalues with positive real part. From Lemma 1 we know that such problems are ill-posed. Furthermore, the factor $\mu$ in the denominator of (53) shows that the rate of the exponential growth can get arbitrarily large as $\mu / \lambda \rightarrow 0$. It is therefore very difficult to compensate for such growth with an artificial dissipation term.

\section{$5 \quad$ Numerical experiments}

For a second order hyperbolic equation, energy conservation ensures that all eigenvalues of the spatial operator are either real and negative, or zero. The same property applies to the discretized problem. To avoid any spurious growth in the numerical solutions, it is therefore important to use a discretization that also satisfies energy conservation. Such a discretization was derived for the 3-D elastic wave equation in Nilsson et al [7]. In the present work, we use the corresponding discretization for the two-dimensional case. This numerical method discretizes the elastic wave equation with a second order accurate, energy conserving, finite difference method on a Cartesian grid with constant grid sizes in space and time. The second order method was recently generalized to fourth order accuracy by Sjogreen and Petersson [8], and we use both the second and forth order methods in the following numerical experiments. Note that our finite difference 
methods are based on solving the elastic wave equation as a second order hyperbolic system using summation by parts operators. These methods are fundamentally different from the commonly used staggered grid method developed by Vireaux [9], Levander [6], and others, which is based on solving the elastic wave equation as a first order hyperbolic system.

\subsection{Surface waves}

To study surface waves using real arithmetic, we are interested in the real part of the eigenfunction (32) corresponding to the generalized eigenvalue

$$
s=i \xi_{0}
$$

Assuming $\omega>0$, the real part of (32) can be written as

$$
\begin{aligned}
\mathbf{u}_{s}(x, y, t) & =e^{-\omega \sqrt{1-\tilde{\xi}_{0}^{2}} x}\left(\begin{array}{c}
\cos \left(\omega\left(y+c_{r} t\right)\right) \\
\sqrt{1-\tilde{\xi}_{0}^{2}} \sin \left(\omega\left(y+c_{r} t\right)\right)
\end{array}\right) \\
& +\left(\frac{\tilde{\xi}_{0}^{2}}{2}-1\right) e^{-\omega \sqrt{1-\tilde{\xi}_{0}^{2} \mu /(2 \mu+\lambda)} x}\left(\begin{array}{c}
\cos \left(\omega\left(y+c_{r} t\right)\right) \\
\sin \left(\omega\left(y+c_{r} t\right)\right) / \sqrt{1-\tilde{\xi}_{0}^{2} \mu /(2 \mu+\lambda)}
\end{array}\right) .
\end{aligned}
$$

Here, we define the Rayleigh phase velocity by

$$
c_{r}=\tilde{\xi}_{0} \sqrt{\mu}
$$

To perform reliable numerical simulations, it is of great interest to know the number of grid points per wave length, $P$, that is required to obtain a certain accuracy in a numerical solution. If the wave length is $L=2 \pi /|\omega|$, we define

$$
P=\frac{L}{h}
$$

We consider a periodic domain in the $y$-direction and choose the computational domain to contain exactly one wave length of the solution. In this investigation we shall keep the wave length fixed at $L=1$, which gives the spatial frequency $\omega=2 \pi$. For simplicity, we set $\lambda=1$ in all numerical experiments. A free surface boundary condition is imposed at $x=0$. We truncate the computational domain at $x=L_{x}$ where we impose an inhomogeneous Dirichlet condition. The boundary data is given by the exact solution (56), which is exponentially small along $x=L_{x}$. For all values of $\lambda / \mu$ (see Table 1), $\kappa_{1} /|\omega|>0.2955$, and we make the influence of the Dirichlet boundary closure small by choosing

$$
L_{x}=10, \quad e^{-2 \pi \sqrt{1-\tilde{\xi}_{0}^{2}} L_{x}} \leq e^{-2 \pi \cdot 0.2955 \cdot 10} \approx 8.5 \cdot 10^{-9}
$$



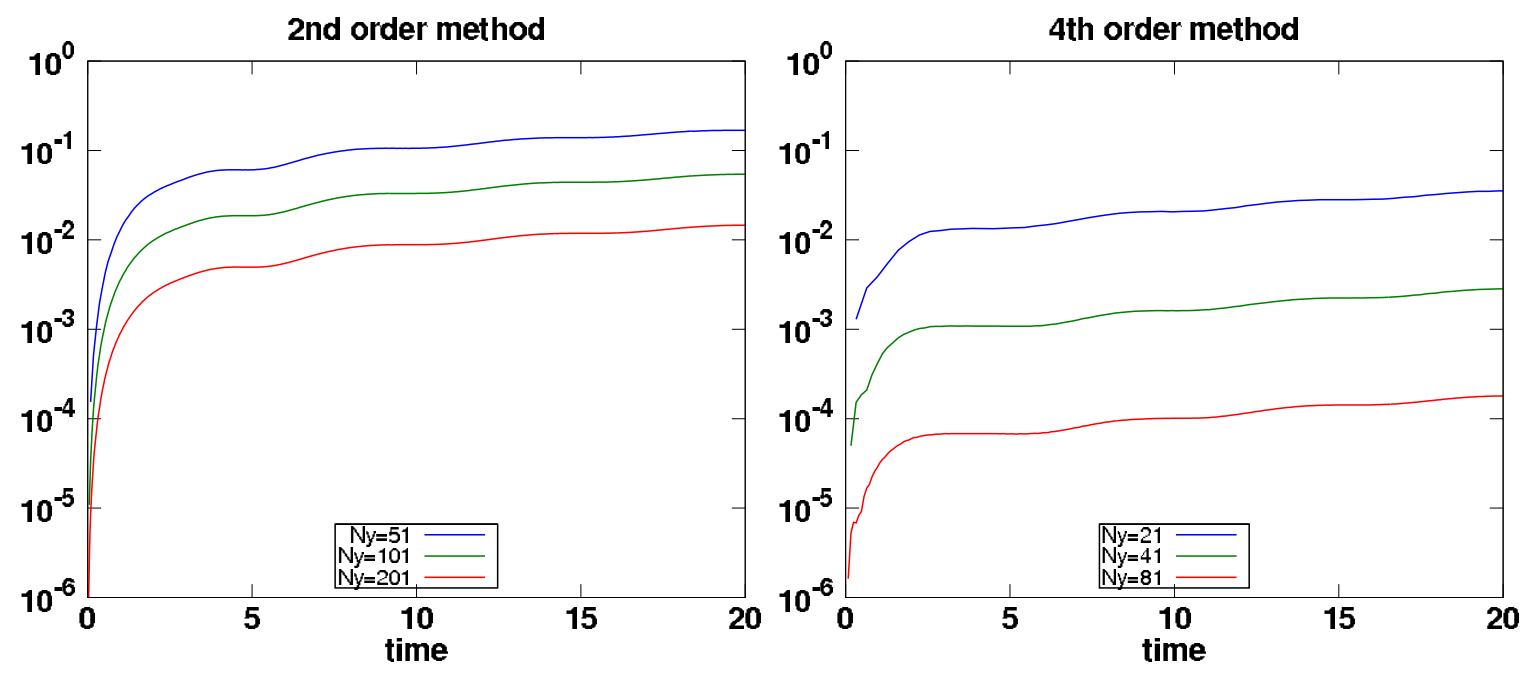

Figure 3: Max error as function of time for a Rayleigh surface wave with $\lambda=1$ and $\mu=0.01$. Results from the second and fourth order methods are shown on the left and right, respectively. The different colors corresponds to different number of grid points per wave length. Note that the grids are coarser for the fourth order computations.

In our first experiment, we take $\mu=0.01$. The numerical solution is evolved from initial data given by (56) at time $t=0$ and $t=-\delta_{t}$, where the time step satisfies the Courant condition (recall that we have scaled time to give unit density)

$$
\delta_{t}=K_{C} \frac{h}{\sqrt{\lambda+3 \mu}}, \quad K_{C}= \begin{cases}0.9, & \text { second order method } \\ 1.3, & \text { fourth order method }\end{cases}
$$

In Figure 3 we show the max norm of the error in the numerical solution as function of time for $t \leq 20$. Since the wave length in the $y$-direction is one, the number of grid points per wave length satisfies $P=N_{y}-1$. Results for the second order accurate method are shown on the left, illustrating the expected convergence rate as the grid is refined. Note that at least 100 grid points per wave length (green line) are needed to obtain a numerical solution to within about $5 \%$ of the exact solution. On the right side of the same figure, we show results for the fourth order method. Here the error decreases by a factor of 16 when the number of grid points is doubled. In this case, only 20 grid points per wave length are needed to make the error less than about $5 \%$ of the exact solution.

In our next experiment, we study how the accuracy depends on $\mu$ when the second order method is used for propagating the Rayleigh wave (56). The period of the wave is

$$
T=\frac{2 \pi}{\omega c_{r}}=\frac{1}{c_{r}}=\frac{1}{\tilde{\xi}_{0} \sqrt{\mu}}
$$




\begin{tabular}{l|c|c|c} 
Case & $P=1 / h$ & $\left\|u_{\text {err }}\right\|_{\infty}(t=T)$ & $\left\|u_{\text {err }}\right\|_{\infty}(t=10 T)$ \\
\hline$\mu=0.1$ & 25 & $5.26 \cdot 10^{-2}$ & $2.99 \cdot 10^{-1}$ \\
$T=3.330$ & 50 & $1.48 \cdot 10^{-2}$ & $9.26 \cdot 10^{-2}$ \\
& 100 & $3.85 \cdot 10^{-3}$ & $2.44 \cdot 10^{-2}$ \\
\hline$\mu=0.01$ & 25 & $2.45 \cdot 10^{-1}$ & $7.64 \cdot 10^{-1}$ \\
$T=10.474$ & 50 & $1.07 \cdot 10^{-1}$ & $5.59 \cdot 10^{-1}$ \\
& 100 & $3.32 \cdot 10^{-2}$ & $2.06 \cdot 10^{-1}$ \\
& 200 & $8.86 \cdot 10^{-3}$ & $5.73 \cdot 10^{-2}$ \\
\hline$\mu=0.001$ & 100 & $1.96 \cdot 10^{-1}$ & $7.69 \cdot 10^{-1}$ \\
$T=33.104$ & 200 & $7.40 \cdot 10^{-2}$ & $4.25 \cdot 10^{-1}$ \\
& 400 & $2.13 \cdot 10^{-2}$ & $1.36 \cdot 10^{-1}$
\end{tabular}

Table 2: The max norm of the error in the numerical evolution of the Rayleigh surface wave, after one and ten periods. Note how the number of grid points per wave length, $P=1 / h$, must be drastically increased to maintain the accuracy as $\mu$ becomes smaller.

In Table 2 we show the max norm of the error after one and ten periods. Note that the period gets longer, i.e., the surface wave propagates slower as $\mu \rightarrow 0$. The case $\mu=0.1$ shows close to second order convergence, both at time $t=T$ and $t=10 T$. The error levels are reasonable for a second order method, but increase with time because the error is dominated by phase errors, i.e., the numerical solution propagates with a slightly different phase velocity compared to the exact solution. The error gets larger for $\mu=0.01$, and a finer grid must used to obtain comparable error levels. For $\mu=0.001$, the grid must be refined further to obtain reasonable error levels, and the cases $P=25$ and $P=50$ are inadequate. A visual inspection shows that after 10 periods, the numerical solution with $P=50$ is more than $180^{\circ}$ out of phase with the exact solution (experiment not shown to save space). We only observe close to second order convergence when the grid is refined from 200 to 400 grid points per wave length.

Note that for $\mu=0.001$, the grid with 200 grid points per wave length gives of the order 10 percent accuracy after one period $\left(\left\|\mathbf{u}_{s}\right\|_{\infty} \approx 0.545\right)$. This grid is about 10 times finer than what is normally required to get that accuracy with a second order method [4]. In the $x$-direction, the gradient of the exact solution is the largest along $x=0$, and $\left|v_{x}\right|=\left|u_{y}\right|$ for all $\mu$. In the limit $\mu \rightarrow 0$, it is straight forward to show $\left|u_{x}\right|=\left|v_{y}\right|$. Hence, the gradient of the exact solution is of the same order in both directions, and conclude that solution is extremely well resolved on the grid. Furthermore, the phase velocity of the surface wave becomes slower and slower as $\mu \rightarrow 0$, while the time step is governed 
by $\sqrt{\lambda+3 \mu}$, which tends to $\sqrt{\lambda}=1$. Hence, the temporal resolution of the surface wave only improves as $\mu \rightarrow 0$.

The analysis of the phase velocity in $\$ 4$ shows that truncation errors in a second order accurate method perturb the generalized eigenvalue according to

$$
\tilde{\xi}=\tilde{\xi}_{0}+\epsilon, \quad \epsilon=\frac{\lambda h^{2} \omega^{2}\left|\alpha_{0}\right|}{\mu} .
$$

The perturbed generalized eigenvalue corresponds to a perturbed phase velocity $c_{r}^{\prime}=\sqrt{\mu} \tilde{\xi}$. Assuming that phase errors dominate the numerical errors, the amplitude of the error follows by

$$
e(t)=\omega\left(c_{r}^{\prime}-c_{r}\right) t=\omega \sqrt{\mu} \epsilon t .
$$

The period of the surface wave follows from (57), so $e(T)=C_{1} \epsilon T, C_{1}=$ const. For a computational grid with grid size $h=1 / P$, (58) gives

$$
\epsilon=\frac{C_{2} \lambda}{P^{2} \mu}, \quad C_{2}=\text { const. }
$$

Hence, to maintain a constant error level in the numerical solution after a fixed number of periods, we must choose $P \sqrt{\mu}=$ const., if $\lambda$ is constant. This assertion is tested by the numerical experiment shown on the left side of Figure 4. Here we show the $\max$ error as function of time scaled by the period of the solution. The first case (red curve) corresponds to $\mu=0.1$, with period $T=3.33$ and resolution $P=40$ grid points per wave length. Notice how closely this error curve follows the case $\mu=10^{-3}$, with period $T=33.104$ and a grid with 400 grid point per wave length. We conclude that the second order method needs a prohibitively fine computational grid to accurately calculate surface waves for small values of $\mu$.

We repeat the above experiment with a fourth order accurate method. The results are shown on the right side of Figure 4. In this case we obtain similar error levels using a significantly coarser grid. For $\mu=0.1$ and $\mu=0.001$, we use $P=12$ and $P=38$, respectively. For the fourth order method, the perturbation of the generalized eigenvalue is given by (55). Using the same argument as for the second order method, we must choose $P \mu^{1 / 4}=$ const. to obtain a constant error level in the numerical solution after a fixed number of periods. This scaling is approximately preserved in these calculations, since

$$
P \mu^{1 / 4} \approx\left\{\begin{array}{l}
6.748, \quad P=12, \mu=0.1 \\
6.757, \quad P=38, \quad \mu=0.001 .
\end{array}\right.
$$

We conclude that the fourth order method is much better suited for simulations when $\mu$ is small. Compared to the second order method, the fourth order method needs a smaller number of grid points per wave length, and the required resolution grows much slower as $\mu \rightarrow 0$. 

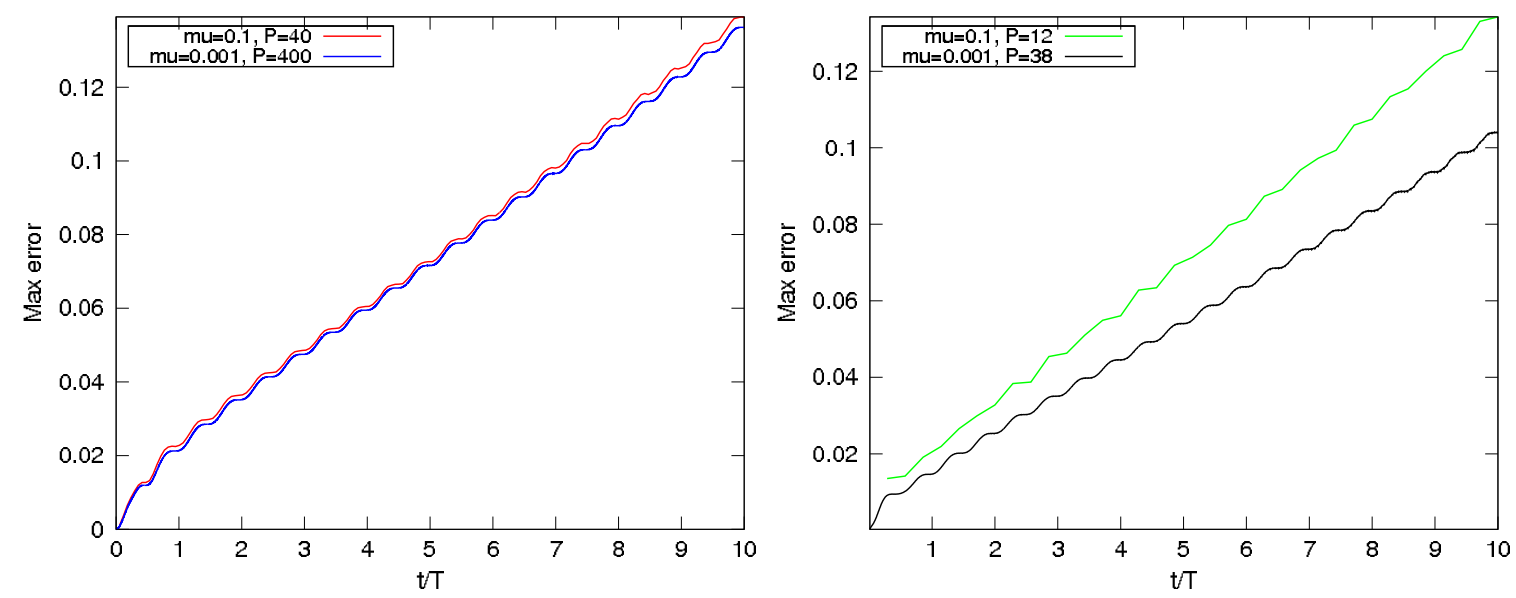

Figure 4: Max error in the numerical evolution of the Rayleigh surface wave, as function of time scaled by the period, $T=1 /\left(\tilde{\xi}_{0} \sqrt{\mu}\right)$. For the second order method (left), the case $\mu=0.1$ with $P=40$ is shown in red and $\mu=0.001$ with $P=400$ is shown in blue. For the fourth order method (right), the case $\mu=0.1$ with $P=12$ (green) and $\mu=0.001$ with $P=38$ (black) give comparable error levels.

To indicate how much more efficient the fourth order method is in practice, we give some execution times obtained on a MacBook Pro laptop computer. The above numerical experiments for $\mu=0.001$ required 20,604 seconds ( $\approx 5$ hours, 43 minutes) for the second order method with $P=400$. Similar accuracy was obtained with the fourth order method using $P=38$, but this calculation only took 60 seconds. Hence, for this problem the fourth order method was 343 times faster than the second order method.

\subsection{Mode to mode conversion}

Consider a compressional wave of unit amplitude traveling in the negative $x$-direction in a homogeneous material, with displacement

$$
\mathbf{u}^{(i n)}=\left(\begin{array}{l}
k \\
\omega
\end{array}\right) e^{i(\xi t+k x+\omega y)}, \quad k=\cos \phi>0, \quad \omega=\sin \phi, \quad \xi>0 .
$$


If this wave encounters a free surface boundary at $x=0$, it will be reflected and split into two waves that both travel in the positive $x$-direction,

$$
\begin{aligned}
\mathbf{u}^{(\text {out })} & =\mathbf{u}^{(P)}+\mathbf{u}^{(S)}, \\
\mathbf{u}^{(P)} & =R_{p}\left(\begin{array}{c}
-k \\
\omega
\end{array}\right) e^{i(\xi t-k x+\omega y)}, \\
\mathbf{u}^{(S)} & =\frac{R_{s}}{\sqrt{\alpha^{2} k^{2}+\omega^{2}}}\left(\begin{array}{c}
-\omega \\
-\alpha k
\end{array}\right) e^{i(\xi t-\alpha k x+\omega y)}, \quad \alpha>0 .
\end{aligned}
$$

The reflected waves correspond to a compressional and a shear wave, since the curl of $\mathbf{u}^{(P)}$ and the divergence of $\mathbf{u}^{(S)}$ are zero. In order for $\mathbf{u}^{(i n)}$ and $\mathbf{u}^{(\text {out })}$ to satisfy the elastic wave equation (1) with $F_{1}=F_{2}=0$, the frequency and wave numbers must satisfy the elementary relations

$$
\xi^{2}=(\lambda+2 \mu)\left(k^{2}+\omega^{2}\right)=\lambda+2 \mu, \quad \xi^{2}=\mu\left(\alpha^{2} k^{2}+\omega^{2}\right)
$$

We select the signs of $\xi$ and $\alpha$ such that $\mathbf{u}^{(i n)}$ and $\mathbf{u}^{(\text {out })}$ travel in the negative and positive $x$-direction, respectively. The amplitudes of the reflected waves, $R_{p}$ and $R_{s}$, are functions of $\lambda, \mu$, and the angle of the incident wave, $\phi$. The amplitudes $R_{p}$ and $R_{s}$ are uniquely determined by the free surface boundary conditions (3) (with $g_{1}=g_{2}=0$ ). For a more detailed discussion, we refer to Achenbach [1], § 5.6.

As a consequence of the relation (59),

$$
\alpha^{2}=1+\frac{\lambda+\mu}{\mu \cos ^{2} \phi} .
$$

Hence, when $\mu \ll \lambda$, the reflected S-wave will propagate almost parallel to the $x$-direction because $\alpha^{2} \gg 1$, see Figure 5. The wave lengths of the compressional and shear waves are given by

$$
L_{p}=\frac{2 \pi}{\sqrt{k^{2}+\omega^{2}}}=2 \pi, \quad L_{s}=2 \pi \sqrt{\frac{\mu}{\lambda+2 \mu}} .
$$

Note that the wave length of the compressional wave is fixed, while $L_{s}$ becomes small as $\mu \rightarrow 0$.

To include two wave lengths of $\mathbf{u}^{(i n)}$ in the computational domain, we take $L_{y}=$ $4 \pi / \sin \phi$ and $L_{x}=4 \pi / \cos \phi$. As before, we impose periodic boundary conditions in the $y$-direction, a Dirichlet boundary condition at $x=L_{x}$ and a free surface condition at $x=0$. By construction, the function $\mathbf{u}^{(i n)}+\mathbf{u}^{(\text {out })}$ is $L_{y}$-periodic in the $y$-direction, satisfies the elastic wave equation in the interior, and the free surface condition at $x=0$. In principle, we could compute a numerical approximation of $\mathbf{u}^{(i n)}+\mathbf{u}^{(\text {out })}$ by adding a suitable forcing function to the Dirichlet boundary condition at $x=L_{x}$. However, we 

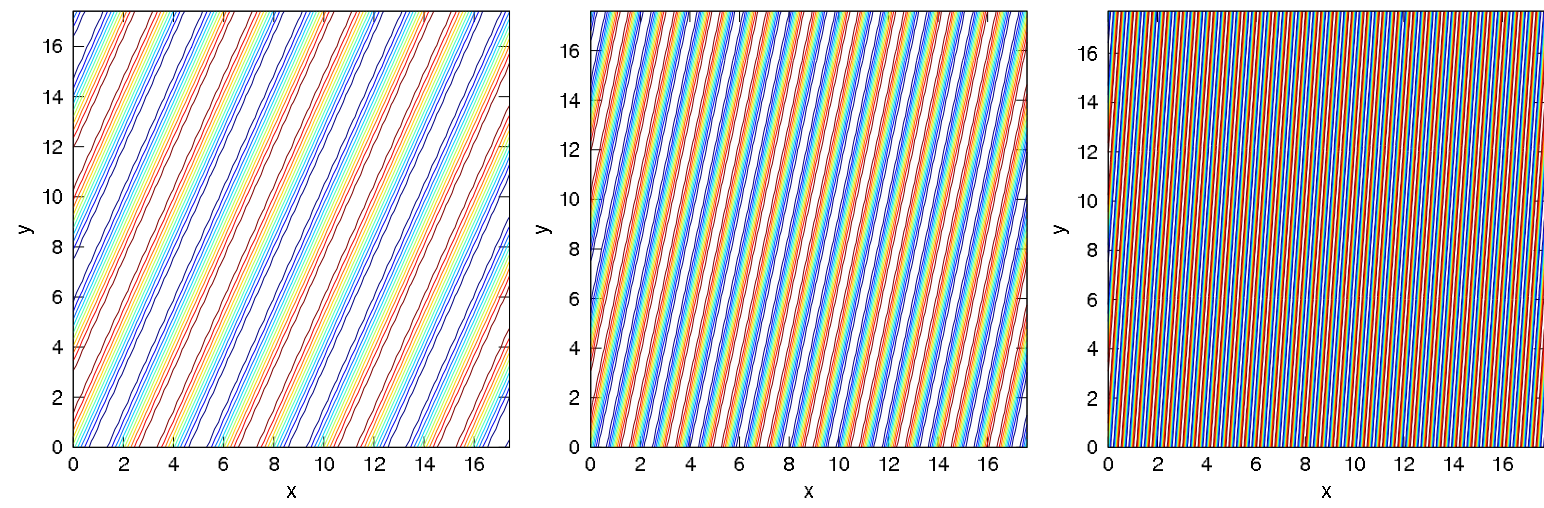

Figure 5: $v$-component of the outgoing shear wave as function of $(x, y)$ at $t=0$. The angle of the incoming $\mathrm{P}$-wave is $\phi=\pi / 4$. The frames correspond to $\mu=1.0$ (left), $\mu=0.1$ (middle), and $\mu=0.01$ (right).

instead choose to only compute the outgoing S-wave, $\mathbf{u}^{(S)}$. For this reason, we impose the inhomogeneous Dirichlet boundary condition

$$
\mathbf{u}\left(L_{x}, y, t\right)=\mathbf{u}^{(S)}\left(L_{x}, y, t\right)
$$

and take the forcing functions in the normal stress boundary conditions (3) to be

$$
\begin{aligned}
& g_{1}=-\left(u_{x}^{(i n)}+u_{x}^{(P)}\right)-\gamma^{2}\left(v_{y}^{(i n)}+v_{y}^{(P)}\right) \\
& g_{2}=-\left(u_{y}^{(i n)}+u_{y}^{(P)}+v_{x}^{(i n)}+v_{x}^{(P)}\right)
\end{aligned}
$$

We use the exact solution $\mathbf{u}^{(S)}$ as initial conditions for the numerical solution.

To accurately solve this problem numerically, it is necessary to resolve the short shear waves on the computational grid. For this problem, we define the resolution in terms of the number of grid points per shear wave length,

$$
P_{s}=\frac{L_{s}}{h}=\frac{\sqrt{\mu}}{h} \frac{2 \pi}{\sqrt{\lambda+2 \mu}}
$$

We evaluate the error in the numerical solution as function of time for two materials. The first material has $(\lambda=1, \mu=0.1)$ and the second has $(\lambda=1, \mu=0.01)$. As a consequence, the period of the wave is slightly different for the two cases

$$
T=\frac{2 \pi}{\xi}=\frac{2 \pi}{\sqrt{\lambda+2 \mu}} \approx \begin{cases}5.74, & \mu=0.1 \\ 6.22, & \mu=0.01\end{cases}
$$

In Figure 6 we show the error as function of normalized time, $t / T$, for the two materials, using the fourth order accurate method. Note that the error levels are comparable for 


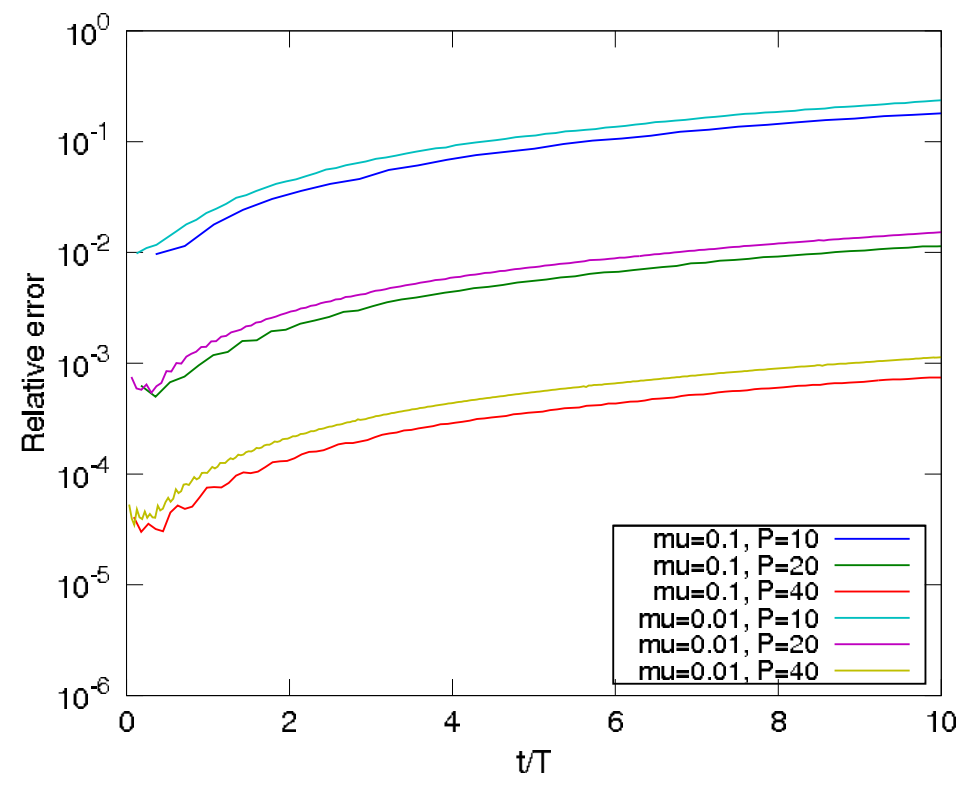

Figure 6: Results for computing the outgoing shear wave with different resolution, characterized by the number of grid points per wave length, $P_{s}$. The relative error in max norm is shown as function of time scaled by the period of the wave. Two cases are shown, $(\lambda=1, \mu=0.1)$ and $(\lambda=1, \mu=0.01)$.

the same number of grid points per wave length, and converge to zero as $\mathcal{O}\left(P_{s}^{-4}\right)$ as the grid is refined. Thus the mode to mode conversion problem does not suffer from the same extreme resolution requirements as the surface wave problem in the previous section. Because we have scaled the problem such that the P-waves have wave length $2 \pi$, the S-waves get a wave length of the order $2 \pi \sqrt{\mu}$. Hence, to keep the number of grid points per S-wave length constant for different materials, we have to choose the grid size according to

$$
h=\frac{2 \pi}{\sqrt{\lambda+2 \mu}} \frac{\sqrt{\mu}}{P_{s}} .
$$

Compared to the material with $\mu=0.1$, the grid size must therefore be taken about a factor of $\sqrt{10}$ smaller for the case $\mu=0.01$, to obtain the same number of grid points per wave length. This scaling is independent of the order of accuracy in the numerical method.

No surface waves can be triggered by a propagating P-wave because the relation (59) shows that $\xi^{2} /\left(\mu \omega^{2}\right)>1$. However, evanescent modes due to an interior forcing function could trigger both S-waves and surface waves. Since the surface waves are only slightly slower than the S-waves, their wave length is of the same order as the length of an S-wave of the same frequency. If the problem is scaled such that the P-wave length is constant, both the S-wave and the surface waves would therefore have wave lengths of the order 
$\sqrt{\mu}$. Based on the results of Section [5.1, a second order accurate method would need a grid size of the order $h \sim \mu$ to maintain a constant accuracy in the numerical solution as $\mu \rightarrow 0$. For a fourth order method, it would suffice to use $h \sim \mu^{3 / 4}$.

\section{Conclusions}

We have developed a normal mode analysis for the half-plane problem of the elastic wave equation subject to a free surface boundary condition. Our analysis allows the solution to be estimated in terms of the boundary data, showing that the solution is as smooth as the boundary forcing. Hence, using the terminology of [5], the problem is boundary stable. The dependence on the material properties is transparent in our estimates. Using a modified equation approach, the normal mode technique was extended to analyze the influence of truncation errors in a finite difference approximation. Our analysis explains why the number of grid points per wave length must be so large when calculating surface waves in materials with $\mu / \lambda \ll 1$. To obtain a fixed error in the phase velocity of Rayleigh surface waves, our analysis predicts that the grid size must be proportional to $\mu^{1 / 2}$ for a second order method, when $\lambda=$ const. For a fourth order method, the analysis shows that it suffices to use $h \sim \mu^{1 / 4}$. These scalings have been confirmed by numerical experiments.

It is theoretically possible to derive stable finite difference schemes that give higher than fourth order accuracy. These methods use wider stencils that are more expensive to evaluate, but for the surface wave problem, it would suffice to use a grid size of the order $h \sim \mu^{1 / p}$, where $p$ is the order of accuracy. For sufficiently small values of $\mu$ these methods should be more efficient as the order of accuracy increases. However, numerical experiments must be performed to evaluate how small $\mu$ must actually be to compensate for the higher computational complexity of these very high order accurate methods.

\section{Acknowledgments}

We thank Tom Hagstrom for discussions that lead to a simple proof of Lemma 6.

\section{A Miscellaneous lemmata}

Lemma 6. Let $\omega$ be a real number and let $s=\eta+i \xi$ be a complex number where $\eta>0$. Consider the relation

$$
\kappa=\sqrt{\omega^{2}+s^{2}},
$$

where the branch cut in the square root is defined by

$$
-\pi<\arg (\alpha+i \beta) \leq \pi, \quad \arg \sqrt{\alpha+i \beta}=\frac{1}{2} \arg (\alpha+i \beta) .
$$


Then,

$$
\operatorname{Re}(\kappa) \geq \eta
$$

Proof. Since $\eta>0$, we can write

$$
s=\eta\left(1+i \xi^{\prime}\right), \quad \kappa=\eta \sqrt{\omega^{\prime 2}+\left(1+i \xi^{\prime}\right)^{2}}, \quad \xi^{\prime}=\frac{\xi}{\eta}, \quad \omega^{\prime}=\frac{\omega}{\eta} .
$$

Define real numbers $a$ and $b$ such that

$$
a+i b=\sqrt{\tilde{\omega}^{2}+\left(1+i \xi^{\prime}\right)^{2}}, \quad a \geq 0 .
$$

Squaring relation (63) and identifying the real and imaginary parts give

$$
\begin{aligned}
a b & =\xi^{\prime}, \\
a^{2}-b^{2} & =1-\xi^{\prime 2}+\omega^{\prime 2} .
\end{aligned}
$$

The first relation gives $b=\xi^{\prime} / a$, which inserted into the second relation results in

$$
a^{2}-\frac{\xi^{\prime 2}}{a^{2}}=1-\xi^{\prime 2}+\omega^{\prime 2} .
$$

Note that the left hand side is a monotonically increasing function of $a^{2}$. When $\omega^{\prime}=0$, equation (64) is solved by $a^{2}=1$. The right hand side of (64) is a monotonically increasing function of $\omega^{\prime 2}$. Therefore $a^{2}>1$ for ${\omega^{\prime 2}}^{2}>0$. We conclude that the unique solution of (64) satisfies

$$
a^{2} \geq 1 \text {. }
$$

Because $a$ must be non-negative, we have $a \geq 1$. Relations (62) and (63) give

$$
\operatorname{Re}(\kappa)=\eta \operatorname{Re} \sqrt{\omega^{\prime 2}+\left(1+i \xi^{\prime}\right)^{2}}=\eta a \geq \eta .
$$

Corollary 1. Let $\omega$ be a real number, $s=\eta+i \xi$ be a complex number with $\eta>0$, and let $0<\gamma<\infty$ be a constant. Then there is another constant $0<\delta<\infty$ such that

$$
\operatorname{Re} \sqrt{\omega^{2}+\frac{s^{2}}{\gamma^{2}}} \geq \delta \eta, \quad \eta=\operatorname{Re}(s)>0 .
$$

Proof. Let $s^{\prime}=s / \gamma$. Lemma 6 proves that

$$
\operatorname{Re} \sqrt{\omega^{2}+\frac{s^{2}}{\gamma^{2}}}=\operatorname{Re} \sqrt{\omega^{2}+s^{\prime 2}} \geq \operatorname{Re}\left(s^{\prime}\right)=\frac{1}{\gamma} \operatorname{Re}(s) .
$$

Hence, $\delta=1 / \gamma>0$ and the corollary follows. 


\section{B The case $\omega \rightarrow 0$}

We now extend the boundary estimates in Section 3.3 to the case $\omega \rightarrow 0$ when $s=s_{0}$, $\left|s_{0}\right|>0$ is fixed. In this limit,

$$
\left|\tilde{s}^{2}\right|=\left|\frac{s^{2}}{\mu \omega^{2}}\right| \rightarrow \infty
$$

For $|\tilde{s}| \gg 1$, we can simplify (37) according to

$$
\tilde{s}^{2} \sqrt{\frac{\mu}{\lambda+2 \mu}} \hat{u}_{01}+\frac{\tilde{s}^{2}}{2} \hat{u}_{02}=-\frac{\lambda+2 \mu}{2 \mu|\omega|} \sqrt{\frac{\mu}{\lambda+2 \mu}} \tilde{s} \hat{g}_{1} .
$$

In a similar way, (38) becomes

$$
\hat{u}_{01}+\frac{2}{\tilde{s}^{2}} \hat{u}_{02}=-\frac{i}{2 \omega \tilde{s}^{2}} \hat{g}_{2} .
$$

Solving the latter equation for $\hat{u}_{01}$ and inserting into (66) gives

$$
\left(-2 \sqrt{\frac{\mu}{\lambda+2 \mu}}+\frac{\tilde{s}^{2}}{2}\right) \hat{u}_{02}=-\frac{(\lambda+2 \mu) \tilde{s} \hat{g}_{1}}{2 \mu|\omega|} \sqrt{\frac{\mu}{\lambda+2 \mu}}+\frac{i \hat{g}_{2}}{2 \omega} \sqrt{\frac{\mu}{\lambda+2 \mu}}
$$

For large $|\tilde{s}|$, we have to leading order,

$$
\hat{u}_{02}=-\sqrt{\frac{\lambda+2 \mu}{\mu}} \frac{\hat{g}_{1}}{|\omega| \tilde{s}}+\sqrt{\frac{\mu}{\lambda+2 \mu}} \frac{i \hat{g}_{2}}{\omega \tilde{s}^{2}}
$$

Note that $s=\tilde{s} \sqrt{\mu}|\omega|$, and

$$
\frac{1}{\tilde{s}|\omega|}=\frac{\sqrt{\mu}}{s}, \quad \frac{1}{\tilde{s}^{2}|\omega|}=\frac{\mu|\omega|}{s^{2}}, \quad \frac{1}{\tilde{s}^{2}}=\frac{\mu \omega^{2}}{s^{2}} .
$$

Hence, (68) and (67) give

$$
\hat{u}_{02}=-\sqrt{\lambda+2 \mu} \frac{\hat{g}_{1}}{s}+\mathcal{O}(\omega), \quad \hat{u}_{01}=-\frac{i \mu \omega \hat{g}_{2}}{2 s^{2}}+\mathcal{O}\left(\omega^{2}\right), \quad \omega \rightarrow 0 .
$$

The solution on the boundary follows from (41) and gives directly

$$
\hat{u}(0)=\hat{u}_{01}+\hat{u}_{02}=-\frac{\sqrt{\lambda+2 \mu} \hat{g}_{1}}{s}+\mathcal{O}(\omega) .
$$

For large $|\tilde{s}|$, we can simplify the expression for $\hat{v}(0)$,

$$
\hat{v}(0)=-\frac{i|\omega| \tilde{s} \hat{u}_{01}}{\omega}-\frac{i \omega \hat{u}_{02}}{|\omega| \tilde{s}} \sqrt{\frac{\lambda+2 \mu}{\mu}}=-\frac{\sqrt{\mu} \hat{g}_{2}}{2 s}+\mathcal{O}(\omega) .
$$

The expressions for $\hat{u}(0)$ and $\hat{v}(0)$ show that the solution is well behaved in the limit $\omega \rightarrow 0$. 


\section{References}

[1] J. D. Achenbach. Wave propagation in elastic solids, volume 16 of Applied Mathematics and Mechanics. North-Holland, 1973.

[2] B. Gustafsson, H.-O. Kreiss, and J. Oliger. Time dependent problems and difference methods. Wiley-Interscience, 1995.

[3] H.-O. Kreiss and J. Lorenz. Initial-Boundary Value Problems and the Navier-Stokes Equations. Academic Press, 1989.

[4] H.-O. Kreiss and J. Oliger. Comparison of accurate methods for the integration of hyperbolic equations. Tellus, 24:199-215, 1972.

[5] H.-O. Kreiss, O.E. Ortiz, and N.A. Petersson. Initial-boundary value problems for second order systems of partial differential equations. LLNL-JRNL 416303, Lawrence Livermore National Laboratory, 2009. To appear in Math. Model. Numer. Anal.

[6] A.R. Levander. Fourth-order finite-difference P-SV seismograms. Geophysics, 53:14251436, 1988.

[7] S. Nilsson, N. A. Petersson, B. Sjögreen, and H.-O. Kreiss. Stable difference approximations for the elastic wave equation in second order formulation. SIAM J. Numer. Anal., 45:1902-1936, 2007.

[8] B. Sjögreen and N. A. Petersson. A fourth order accurate finite difference scheme for the elastic wave equation in second order formulation. Technical report, Lawrence Livermore National Laboratory, 2011. To be submitted.

[9] J. Virieux. P-SV wave propagation in heterogeneous media: Velocity-stress finitedifference method. Geophysics, 51:889-901, 1986. 